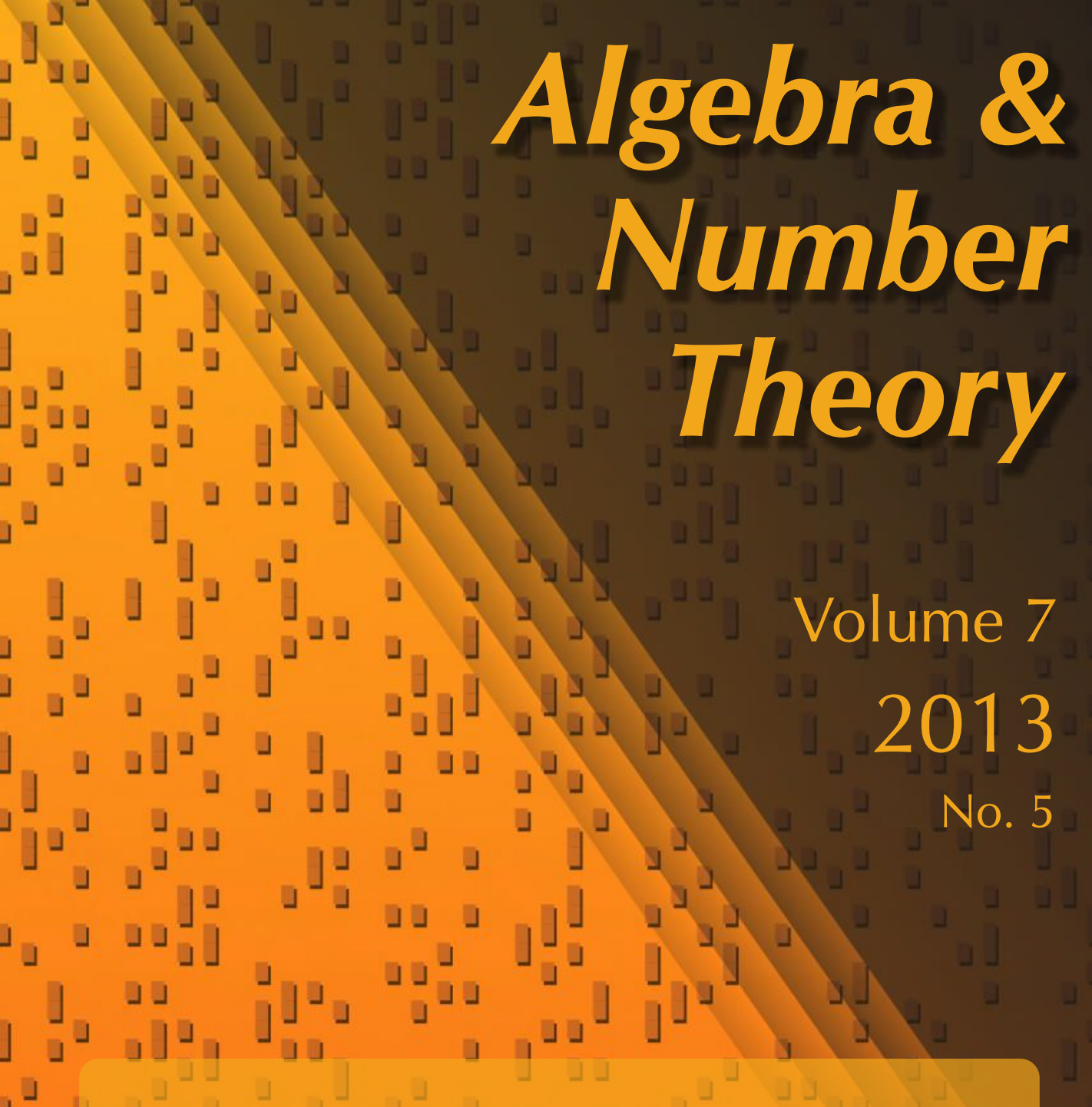

Quantized mixed tensor space and Schur-Weyl duality

Richard Dipper, Stephen Doty and Friederike Stoll

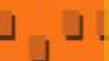

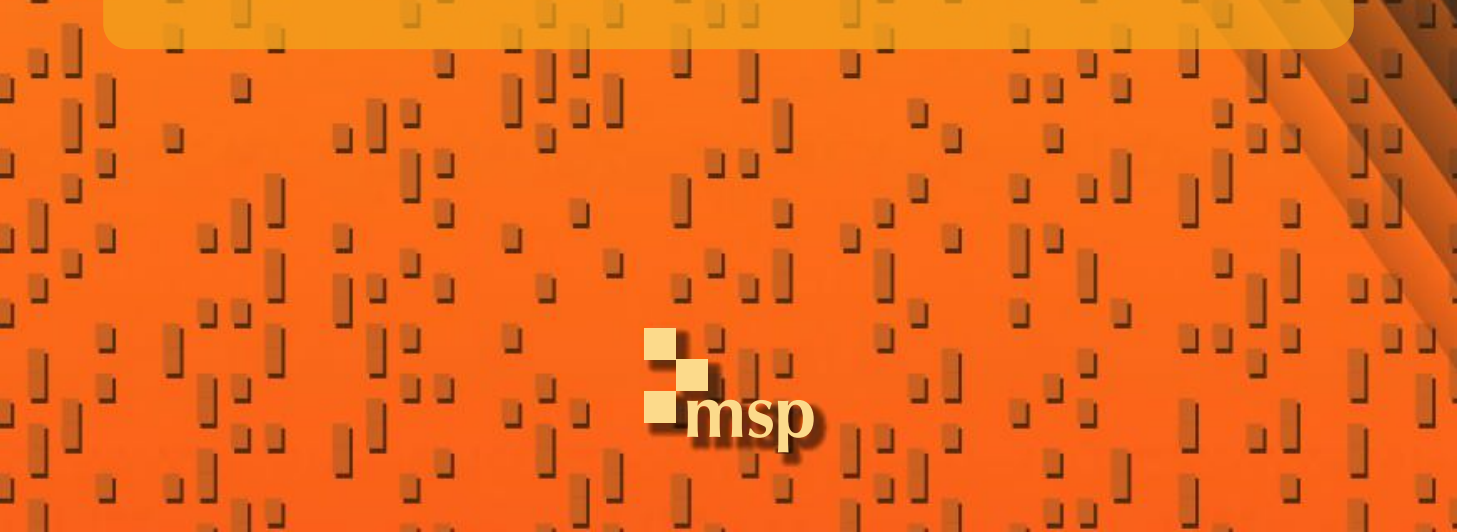




\title{
Quantized mixed tensor space and Schur-Weyl duality
}

\author{
Richard Dipper, Stephen Doty and Friederike Stoll
}

Let $R$ be a commutative ring with 1 and $q$ an invertible element of $R$. The (specialized) quantum group $\mathbf{U}=U_{q}\left(\mathfrak{g l}_{n}\right)$ over $R$ of the general linear group acts on mixed tensor space $V^{\otimes r} \otimes V^{* \otimes s}$, where $V$ denotes the natural $\mathbf{U}$-module $R^{n}, r$ and $s$ are nonnegative integers and $V^{*}$ is the dual $\mathbf{U}$-module to $V$. The image of $\mathbf{U}$ in $\operatorname{End}_{R}\left(V^{\otimes r} \otimes V^{* \otimes s}\right)$ is called the rational $q$-Schur algebra $S_{q}(n ; r, s)$. We construct a bideterminant basis of $S_{q}(n ; r, s)$. There is an action of a $q$-deformation $\mathfrak{B}_{r, s}^{n}(q)$ of the walled Brauer algebra on mixed tensor space centralizing the action of $\mathbf{U}$. We show that $\operatorname{End}_{\mathfrak{B}_{r, s}^{n}(q)}\left(V^{\otimes r} \otimes V^{* \otimes s}\right)=S_{q}(n ; r, s)$. By a previous result, the image of $\mathfrak{B}_{r, s}^{n}(q)$ in $\operatorname{End}_{R}\left(V^{\otimes r} \otimes V^{* \otimes s}\right)$ is $\operatorname{End}_{\mathbf{U}}\left(V^{\otimes r} \otimes V^{* \otimes s}\right)$. Thus, a mixed tensor space as $\left(\mathbf{U}, \mathfrak{B}_{r, s}^{n}(q)\right)$-bimodule satisfies Schur-Weyl duality.

\section{Introduction}

Schur-Weyl duality plays an important role in representation theory since it relates the representations of the general linear group with the representations of the symmetric group. The classical Schur-Weyl duality, due to Schur [1927], states that the actions of the general linear group $G=\mathrm{GL}_{n}(\mathbb{C})$ and the symmetric group $\mathfrak{S}_{m}$ on the tensor space $V^{\otimes m}$ with $V=\mathbb{C}^{n}$ satisfy the bicentralizer property, that is, $\operatorname{End}_{\mathfrak{S}_{m}}\left(V^{\otimes m}\right)$ is generated by the action of $G$ and correspondingly, $\operatorname{End}_{G}\left(V^{\otimes m}\right)$ is generated by the action of $\mathfrak{S}_{m}$. This duality has been generalized to subgroups of $G$ (e.g., orthogonal, symplectic groups, and Levi subgroups) and corresponding algebras related with the group algebra of the symmetric group (e.g., Brauer algebras and Ariki-Koike algebras) as well as deformations of these algebras. In general, the phrase "Schur-Weyl duality" has come to indicate such a bicentralizer property for two algebras acting on some module.

One such generalization is the mixed tensor space $V^{\otimes r} \otimes V^{* \otimes s}$, where $V$ is the natural and $V^{*}$ its dual $\mathbb{C} G$-module. The centralizer algebra is known to be the walled Brauer algebra $\mathfrak{B}_{r, s}^{n}$, and it was shown by Benkart, Chakrabarti, Halverson, Leduc, Lee and Stroomer [Benkart et al. 1994] that mixed tensor space under the

MSC2010: primary 33D80; secondary 16D20, 16S30, 17B37, $20 \mathrm{C} 08$.

Keywords: Schur-Weyl duality, walled Brauer algebra, mixed tensor space, rational $q$-Schur algebra. 
action of $\mathbb{C} G$ and $\mathfrak{B}_{r, s}^{n}$ satisfies Schur-Weyl duality; see also [Koike 1989; Turaev 1989]. In [Kosuda and Murakami 1993] the authors introduced a one-parameter deformation $\mathfrak{B}_{r, s}^{n}(q)$ of the walled Brauer algebra and proved Schur-Weyl duality in the generic case (i.e., over $\mathbb{C}(q)$ ), where $\mathbb{C} G$ is replaced by the generic quantum group $U_{\mathbb{C}(q)}\left(\mathfrak{g l}_{n}\right)$.

In this paper, we generalize the results of [Benkart et al. 1994; Kosuda and Murakami 1993] to a very general setting. Let $R$ be a commutative ring with 1 and $q \in R$ be invertible. Let $\mathbf{U}$ be (a specialized version of) the quantum group over $R$, which replaces the general linear group in the quantized case. Let $\mathfrak{B}_{r, s}^{n}(q)$ be the $q$-deformation of the walled Brauer algebra defined in [Leduc 1994]. Here we use a specialized version of Leduc's multiparameter version that acts on mixed tensor space $V^{\otimes r} \otimes V^{* \otimes s}$, where $V=R^{n}$ is the natural $\mathbf{U}$-module.

In [Dipper et al. 2012], one side of Schur-Weyl duality was shown in this situation, namely that the image of $\mathfrak{B}_{r, s}^{n}(q)$ in $\operatorname{End}_{R}\left(V^{\otimes r} \otimes V^{* \otimes s}\right)$ is the centralizing algebra of the action of $\mathbf{U}$ on mixed tensor space.

In this paper, which is a revised version of a preprint that has circulated since 2008 , the other side of Schur-Weyl duality will be proven, namely that the image of $\mathbf{U}$ in $\operatorname{End}_{R}\left(V^{\otimes r} \otimes V^{* \otimes s}\right)$ is the endomorphism algebra of mixed tensor space under the action of $\mathfrak{B}_{r, s}^{n}(q)$. We call this image the rational $q$-Schur algebra and denote it $S_{q}(n ; r, s)$. It is a $q$-analogue of the rational Schur algebra introduced and studied in [Dipper and Doty 2008]. In case $q=1$, we obtain a similar statement (which is also new) for the rational Schur algebra with respect to the hyperalgebra over $R$ of $\mathfrak{g l}_{n}$. In the meantime, this result was shown in [Tange 2012] in the special case $q=1$ by different methods. One may also wish to consult [Brundan and Stroppel 2011], which enlarges the landscape on walled Brauer algebras considerably.

For technical reasons, it will be useful to turn things around and instead define $S_{q}(n ; r, s)$ to be $\operatorname{End}_{\mathfrak{B}_{r, s}^{n}(q)}\left(V^{\otimes r} \otimes V^{* \otimes s}\right)$. Since we show at the end that this coincides with the image of $\mathbf{U}$ in $\operatorname{End}_{R}\left(V^{\otimes r} \otimes V^{* \otimes s}\right)$, there is no harm in this abuse of notation. In our proof, we will show that $\operatorname{End}_{\mathfrak{B}_{r, s}^{n}(q)}\left(V^{\otimes r} \otimes V^{* \otimes s}\right)=S_{q}(n ; r, s)$ is free as $R$-module of rank independent of the choice of $R$ and $q$. We shall accomplish this by constructing an $R$-basis of $S_{q}(n ; r, s)$ that is dual to a certain bideterminant basis of the dual coalgebra $A_{q}(n ; r, s)$ of $S_{q}(n ; r, s)$.

As a guide for the reader, we briefly outline the main ideas behind the proof. There is a natural embedding of mixed tensor space $V^{\otimes r} \otimes V^{* \otimes s}$ into ordinary tensor space $V^{\otimes r+(n-1) s}$. This embedding $\kappa$ is not $\mathbf{U}$-linear but is $\mathbf{U}^{\prime}$-linear, where $\mathbf{U}^{\prime}$ is the subalgebra of $\mathbf{U}$ corresponding to the special linear Lie algebra. We will see that replacing $\mathbf{U}$ by $\mathbf{U}^{\prime}$ is not significant. For $u \in \mathbf{U}^{\prime}$, the restriction of the action of $u$ on $V^{\otimes r+(n-1) s}$ to $V^{\otimes r} \otimes V^{* \otimes s} \leq V^{\otimes r+(n-1) s}$ commutes with the action of $\mathfrak{B}_{r, s}^{n}(q)$ on $V^{\otimes r} \otimes V^{* \otimes s}$ and hence lies in $S_{q}(n ; r, s)$. Thus, $\kappa$ induces an algebra homomorphism $\pi$ from the ordinary $q$-Schur algebra $S_{q}(n, r+(n-1) s)$, which 
is the image of $\mathbf{U}^{\prime}$ in $\operatorname{End}_{R}\left(V^{\otimes r+(n-1) s}\right)$ into $S_{q}(n ; r, s)$. This homomorphism was motivated by a similar homomorphism in [Dipper and Doty 2008].

Let $\rho_{\text {ord }}: \mathbf{U}^{\prime} \rightarrow S_{q}(n, r+(n-1) s)$ be the representation of $\mathbf{U}^{\prime}$ on $V^{\otimes r+(n-1) s}$ and $\rho_{\mathrm{mxd}}: \mathbf{U}^{\prime} \rightarrow S_{q}(n ; r, s)$ the representation of $\mathbf{U}^{\prime}$ on mixed tensor space. Then $\rho_{\mathrm{mxd}}=\pi \circ \rho_{\text {ord }}$ by construction. By classical quantized Schur-Weyl duality, $\rho_{\text {ord }}$ is surjective, so $\rho_{\mathrm{mxd}}$ is surjective (i.e., $\left.\rho_{\mathrm{mxd}}\left(\mathbf{U}^{\prime}\right)=S_{q}(n ; r, s)\right)$ if $\pi$ is surjective. We show that $\pi$ possesses an $R$-linear right inverse, thus proving the surjectivity of $\pi$.

At this point, we switch over to coefficient spaces. It is well known that the dual coalgebra $A_{q}(n, r+(n-1) s)=S_{q}(n, r+(n-1) s)^{*}$ is the coefficient space of $\mathbf{U}^{\prime}$ acting on ordinary tensor space $V^{\otimes r+(n-1) s}$. There is no problem here with dualization since the classical $q$-Schur algebra $S_{q}(n, r+(n-1) s)$ is known to be free as $R$-module of fixed rank independent of the choice of $R$ and $q$. Moreover, $A_{q}(n, r+(n-1) s)$ possesses a bideterminant basis [Huang and Zhang 1993]. The endomorphism algebra $S_{q}(n ; r, s)=\operatorname{End}_{\mathfrak{B}_{r, s}^{n}(q)}\left(V^{\otimes r} \otimes V^{* \otimes s}\right)$ may be described by a system of linear equations in the endomorphism algebra $\operatorname{End}_{R}\left(V^{\otimes r} \otimes V^{* \otimes s}\right)$, which is free as $R$-module. Using these equations, we apply a general argument (Lemma 2.3) to construct a factor coalgebra $A_{q}(n ; r, s)$ of the $R$-coalgebra $\operatorname{End}_{R}\left(V^{\otimes r} \otimes V^{* \otimes s}\right)$ such that $A_{q}(n ; r, s)^{*}$ is isomorphic to the $R$-algebra $S_{q}(n ; r, s)$. In Section 5, we exhibit a map $\iota: A_{q}(n ; r, s) \rightarrow A_{q}(n, r+(n-1) s)$ and show explicitly that $\iota^{*}=\pi: S_{q}(n, r+(n-1) s) \rightarrow S_{q}(n ; r, s)$. In Section 6, we show that $A_{q}(n ; r, s)$ and hence $S_{q}(n ; r, s)$ are free as $R$-module by constructing a (rational) bideterminant basis. From this it is not hard to find an ( $R$-linear) left inverse of the map $\iota$ whose dual map is then the required right inverse of $\iota^{*}=\pi$, proving that $S_{q}(n ; r, s)$ is the image of $\mathbf{U}^{\prime}$ (and hence $\mathbf{U}$ ) acting on mixed tensor space.

\section{Preliminaries}

Let $n$ be a given positive integer. In this section, we introduce the quantized enveloping algebra of the general linear Lie algebra $\mathfrak{g l}_{n}$ over a commutative ring $R$ with parameter $q$ and summarize some well known results; see for example [Hong and Kang 2002; Jantzen 1996; Lusztig 1990]. We will start by recalling the definition of the quantized enveloping algebra over $\mathbb{Q}(q)$, where $q$ is an indeterminate.

Let $P^{\vee}$ be the free $\mathbb{Z}$-module with basis $h_{1}, \ldots, h_{n}$, and let $\varepsilon_{1}, \ldots, \varepsilon_{n} \in P^{\vee *}$ be the corresponding dual basis: $\varepsilon_{i}$ is given by $\varepsilon_{i}\left(h_{j}\right):=\delta_{i, j}$ for $j=1, \ldots, n$, where $\delta$ is the usual Kronecker symbol. For $i=1, \ldots, n-1$, let $\alpha_{i} \in P^{\vee *}$ be defined by $\alpha_{i}:=\varepsilon_{i}-\varepsilon_{i+1}$.

Definition 1.1. The quantum general linear algebra $U_{q}\left(\mathfrak{g l}_{n}\right)$ is the associative $\mathbb{Q}(q)$ algebra with 1 generated by the elements $e_{i}, f_{i}(i=1, \ldots, n-1)$ and $q^{h}\left(h \in P^{\vee}\right)$ with the defining relations

$$
q^{0}=1, \quad q^{h} q^{h^{\prime}}=q^{h+h^{\prime}}, \quad q^{h} e_{i} q^{-h}=q^{\alpha_{i}(h)} e_{i}, \quad q^{h} f_{i} q^{-h}=q^{-\alpha_{i}(h)} f_{i},
$$




$$
\begin{gathered}
e_{i} f_{j}-f_{j} e_{i}=\delta_{i, j} \frac{K_{i}-K_{i}^{-1}}{q-q^{-1}}, \quad \text { where } K_{i}:=q^{h_{i}-h_{i+1}}, \\
e_{i}^{2} e_{j}-\left(q+q^{-1}\right) e_{i} e_{j} e_{i}+e_{j} e_{i}^{2}=0 \text { for }|i-j|=1, \\
f_{i}^{2} f_{j}-\left(q+q^{-1}\right) f_{i} f_{j} f_{i}+f_{j} f_{i}^{2}=0 \text { for }|i-j|=1, \\
e_{i} e_{j}=e_{j} e_{i} \quad \text { and } f_{i} f_{j}=f_{j} f_{i} \quad \text { for }|i-j|>1 .
\end{gathered}
$$

We note that the subalgebra generated by the $K_{i}, e_{i}$ and $f_{i}(i=1, \ldots, n-1)$ is isomorphic with $U_{q}\left(\mathfrak{s l}_{n}\right)$. Also, $U_{q}\left(\mathfrak{g l}_{n}\right)$ is a Hopf algebra with comultiplication $\Delta$, counit $\varepsilon$ the unique algebra homomorphisms and antipode $S$ the unique invertible antihomomorphism of algebras, defined on generators by

$$
\begin{gathered}
\Delta\left(q^{h}\right)=q^{h} \otimes q^{h}, \\
\Delta\left(e_{i}\right)=e_{i} \otimes K_{i}^{-1}+1 \otimes e_{i}, \quad \Delta\left(f_{i}\right)=f_{i} \otimes 1+K_{i} \otimes f_{i}, \\
\varepsilon\left(q^{h}\right)=1, \quad \varepsilon\left(e_{i}\right)=\varepsilon\left(f_{i}\right)=0, \\
S\left(q^{h}\right)=q^{-h}, \quad S\left(e_{i}\right)=-e_{i} K_{i}, \quad S\left(f_{i}\right)=-K_{i}^{-1} f_{i} .
\end{gathered}
$$

Let $V_{\mathbb{Q}(q)}$ be a free $\mathbb{Q}(q)$-vector space with basis $\left\{v_{1}, \ldots, v_{n}\right\}$. We make $V_{\mathbb{Q}(q)}$ a $U_{q}\left(\mathfrak{g l}_{n}\right)$-module via

$$
\begin{gathered}
q^{h} v_{j}=q^{\varepsilon_{j}(h)} v_{j} \quad \text { for } h \in P^{\vee} \text { and } j=1, \ldots, n, \\
e_{i} v_{j}=\left\{\begin{array}{ll}
v_{i} & \text { if } j=i+1, \\
0 & \text { otherwise, }
\end{array} \quad f_{i} v_{j}= \begin{cases}v_{i+1} & \text { if } j=i, \\
0 & \text { otherwise. }\end{cases} \right.
\end{gathered}
$$

We call $V_{\mathbb{Q}(q)}$ the vector representation of $U_{q}\left(\mathfrak{g l}_{n}\right)$. This is also a $U_{q}\left(\mathfrak{s l}_{n}\right)$-module by restriction of the action.

Let $[l]_{q}$ in $\mathbb{Z}\left[q, q^{-1}\right]$ (or in $R$ ) be defined by

$$
[l]_{q}:=\sum_{i=0}^{l-1} q^{2 i-l+1}
$$

and set $[l]_{q} !:=[l]_{q}[l-1]_{q} \cdots[1]_{q}$. Define the divided powers $e_{i}^{(l)}:=e_{i}^{l} /[l]_{q}$ ! and $f_{i}^{(l)}:=f_{i}^{l} /[l]_{q}$ !. Let $\mathbf{U}_{\mathbb{Z}\left[q, q^{-1}\right]}\left(\right.$ resp. $\left.\mathbf{U}_{\mathbb{Z}\left[q, q^{-1}\right]}^{\prime}\right)$ be the $\mathbb{Z}\left[q, q^{-1}\right]$-subalgebra of $U_{q}\left(\mathfrak{g l}_{n}\right)$ generated by the $q^{h}$ (resp. the $\left.K_{i}\right)$ and the $e_{i}^{(l)}$ and $f_{i}^{(l)}$ for $l \geq 0$. Then $\mathbf{U}_{\mathbb{Z}\left[q, q^{-1}\right]}$ is a Hopf algebra, and we have

$$
\begin{gathered}
\Delta\left(e_{i}^{(l)}\right)=\sum_{k=0}^{l} q^{k(l-k)} e_{i}^{(l-k)} \otimes K_{i}^{k-l} e_{i}^{(k)}, \quad \Delta\left(f_{i}^{(l)}\right)=\sum_{k=0}^{l} q^{-k(l-k)} f_{i}^{(l-k)} K_{i}^{k} \otimes f_{i}^{(k)}, \\
S\left(e_{i}^{(l)}\right)=(-1)^{l} q^{l(l-1)} e_{i}^{(l)} K_{i}^{l}, \quad S\left(f_{i}^{(l)}\right)=(-1)^{l} q^{-l(l-1)} K_{i}^{-l} f_{i}^{(l)}, \\
\varepsilon\left(e_{i}^{(l)}\right)=\varepsilon\left(f_{i}^{(l)}\right)=0 .
\end{gathered}
$$


Furthermore, the $\mathbb{Z}\left[q, q^{-1}\right]$-lattice $V_{\mathbb{Z}\left[q, q^{-1}\right]}$ in $V_{\mathbb{Q}(q)}$ generated by the $v_{i}$ is invariant under the action of $\mathbf{U}_{\mathbb{Z}\left[q, q^{-1}\right]}$ and of $\mathbf{U}_{\mathbb{Z}\left[q, q^{-1}\right]}^{\prime}$. Now, make the transition from $\mathbb{Z}\left[q, q^{-1}\right]$ to an arbitrary commutative ring $R$ with 1 . Let $q \in R$ be invertible, and consider $R$ as a $\mathbb{Z}\left[q, q^{-1}\right]$-module via specializing $q \in \mathbb{Z}\left[q, q^{-1}\right] \mapsto q \in R$.

Let $\mathbf{U}_{R}:=R \otimes_{\mathbb{Z}\left[q, q^{-1}\right]} \mathbf{U}_{\mathbb{Z}\left[q, q^{-1}\right]}$ and $\mathbf{U}_{R}^{\prime}:=R \otimes_{\mathbb{Z}\left[q, q^{-1}\right]} \mathbf{U}_{\mathbb{Z}\left[q, q^{-1}\right]}^{\prime}$. Then $\mathbf{U}_{R}$ inherits a Hopf algebra structure from $\mathbf{U}_{\mathbb{Z}\left[q, q^{-1}\right]}$, and $V_{R}:=R \otimes_{\mathbb{Z}\left[q, q^{-1}\right]} V_{\mathbb{Z}\left[q, q^{-1}\right]}$ is a $\mathbf{U}_{R}$-module and by restriction also a $\mathbf{U}_{R}^{\prime}$-module.

If no ambiguity arises, we will henceforth omit the index $R$ and write $\mathbf{U}, \mathbf{U}^{\prime}$ and $V$ instead of $\mathbf{U}_{R}, \mathbf{U}_{R}^{\prime}$ and $V_{R}$. Furthermore, we will write $e_{i}^{(l)}$ as shorthand for $1 \otimes e_{i}^{(l)} \in \mathbf{U}_{R}$, similarly for the $f_{i}^{(l)}, K_{i}$ for $1 \otimes K_{i}$ and $q^{h}$ for $1 \otimes q^{h}$.

Suppose $W, W_{1}$ and $W_{2}$ are $\mathbf{U}$-modules; then one can define $\mathbf{U}$-module structures on $W_{1} \otimes W_{2}=W_{1} \otimes_{R} W_{2}$ and $W^{*}=\operatorname{Hom}_{R}(W, R)$ using the comultiplication and the antipode by setting $x\left(w_{1} \otimes w_{2}\right)=\Delta(x)\left(w_{1} \otimes w_{2}\right)$ and $(x f)(w)=f(S(x) w)$.

Definition 1.2. Let $r$ and $s$ be nonnegative integers. The $\mathbf{U}$-module $V^{\otimes r} \otimes V^{* \otimes s}$ is called mixed tensor space.

Let $I(n, r)$ be the set of $r$-tuples with entries in $\{1, \ldots, n\}$, and let $I(n, s)$ be defined similarly. The elements of $I(n, r)$ (and $I(n, s)$ ) are called multi-indices. Note that the symmetric groups $\mathfrak{S}_{r}$ and $\mathfrak{S}_{s}$ act on $I(n, r)$ and $I(n, s)$ respectively from the right by place permutation, that is, if $\boldsymbol{i}=\left(i_{1}, i_{2}, \ldots\right)$ is a multi-index and $s_{j}$ is a Coxeter generator, then let $\mathbf{i} . s_{j}:=\left(i_{1}, \ldots, i_{j-1}, i_{j+1}, i_{j}, i_{j+2}, \ldots\right)$. Then a basis of the mixed tensor space $V^{\otimes r} \otimes V^{* \otimes s}$ can be indexed by $I(n, r) \times I(n, s)$. For $\boldsymbol{i}=\left(i_{1}, \ldots, i_{r}\right) \in I(n, r)$ and $\boldsymbol{j}=\left(j_{1}, \ldots, j_{s}\right) \in I(n, s)$, let

$$
v_{i \mid \mathbf{j}}:=v_{i_{1}} \otimes \cdots \otimes v_{i_{r}} \otimes v_{j_{1}}^{*} \otimes \cdots \otimes v_{j_{s}}^{*} \in V^{\otimes r} \otimes V^{* \otimes s},
$$

where $\left\{v_{1}^{*}, \ldots, v_{n}^{*}\right\}$ is the basis of $V^{*}$ dual to $\left\{v_{1}, \ldots, v_{n}\right\}$. Then $\left\{v_{i \mid j}: \boldsymbol{i} \in I(n, r)\right.$, $\boldsymbol{j} \in I(n, s)\}$ is a basis of $V^{\otimes r} \otimes V^{* \otimes s}$.

We have another algebra acting on $V^{\otimes r} \otimes V^{* \otimes s}$, namely the quantized walled Brauer algebra $\mathfrak{B}_{r, s}^{n}(q)$ introduced in [Dipper et al. 2012]. This algebra is defined as a diagram algebra in terms of Kauffman's tangles. A presentation by generators and relations can be found in [Dipper et al. 2012]. Note that this algebra and its action coincide with Leduc's algebra [1994] (see the remarks in [Dipper et al. 2012]).

Here, all we need is the action of generators given in the following diagrams. The Brauer algebra $\mathfrak{B}_{r, s}^{n}(q)$ is generated by the elements

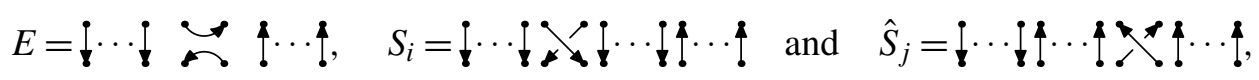

where the nonpropagating edges in $E$ connect vertices in columns $r$ and $r+1$ while the crossings in $S_{i}$ and $\hat{S}_{j}$ connect vertices in columns $i$ and $i+1$ and columns $r+j$ and $r+j+1$, respectively. If $v_{i \mid j}=v \otimes v_{i_{r}} \otimes v_{j_{1}}^{*} \otimes v^{\prime}$, then the action of the 
generators on $V^{\otimes r} \otimes V^{* \otimes s}$ is given by

$$
\begin{aligned}
& v_{i \mid j} E=\delta_{i_{r}, j_{1}} \sum_{s=1}^{n} q^{2 i_{r}-n-1} v \otimes v_{s} \otimes v_{s}^{*} \otimes v^{\prime}, \\
& v_{i \mid j} S_{i}= \begin{cases}q^{-1} v_{i \mid j} & \text { if } i_{i}=i_{i+1}, \\
v_{i . s_{i} \mid j} & \text { if } i_{i}<i_{i+1}, \\
v_{i . s_{i} \mid j}+\left(q^{-1}-q\right) v_{i \mid j} & \text { if } i_{i}>i_{i+1},\end{cases} \\
& v_{i \mid j} \hat{S}_{j}= \begin{cases}q^{-1} v_{i \mid j} & \text { if } j_{j}=j_{j+1}, \\
v_{i \mid j . s_{j}} & \text { if } j_{j}>j_{j+1}, \\
v_{i \mid j . s_{j}}+\left(q^{-1}-q\right) v_{i \mid j} & \text { if } j_{j}<j_{j+1} .\end{cases}
\end{aligned}
$$

The action of $\mathfrak{B}_{r, s}^{n}(q)$ on $V^{\otimes r} \otimes V^{* \otimes s}$ commutes with the action of $\mathbf{U}$.

Theorem 1.3 [Dipper et al. 2012]. Let $\sigma: \mathfrak{B}_{r, s}^{n}(q) \rightarrow \operatorname{End}_{\mathbf{U}}\left(V^{\otimes r} \otimes V^{* \otimes s}\right)$ be the representation of the quantized walled Brauer algebra on the mixed tensor space. Then $\sigma$ is surjective, that is,

$$
\operatorname{End}_{\mathbf{U}}\left(V^{\otimes r} \otimes V^{* \otimes s}\right) \cong \mathfrak{B}_{r, s}^{n}(q) / \operatorname{ann}_{\mathfrak{B}_{r, s}^{n}(q)}\left(V^{\otimes r} \otimes V^{* \otimes s}\right) .
$$

The main result of this paper is the other half of the preceding theorem.

Theorem 1.4. Let $\rho_{\mathrm{mxd}}: \mathbf{U} \rightarrow \operatorname{End}_{\mathfrak{B}_{r, s}^{n}(q)}\left(V^{\otimes r} \otimes V^{* \otimes s}\right)$ be the representation of the quantum group. Then $\rho_{\mathrm{mxd}}$ is surjective, that is,

$$
\operatorname{End}_{\mathfrak{B}_{r, s}^{n}(q)}\left(V^{\otimes r} \otimes V^{* \otimes s}\right) \cong \mathbf{U} / \operatorname{ann}_{\mathbf{U}}\left(V^{\otimes r} \otimes V^{* \otimes s}\right) .
$$

Theorems 1.3 and 1.4 together state that the mixed tensor space is a $\left(\mathbf{U}, \mathfrak{B}_{r, s}^{n}(q)\right)$ bimodule with the double centralizer property. In the literature, this is also called Schur-Weyl Duality. Theorem 1.4 will be proved at the end of this paper.

For $s=0$, this is well known; $\mathfrak{B}_{m, 0}^{n}(q)$ is the Hecke algebra $\mathscr{H}_{m}$, and $V^{\otimes m}$ is the (ordinary) tensor space.

Definition 1.5. If $m$ is a positive integer, let $\mathscr{H}_{m}$ be the associative $R$-algebra with 1 generated by elements $T_{1}, \ldots, T_{m-1}$ with respect to the relations

$$
\begin{aligned}
& \left(T_{i}+q\right)\left(T_{i}-q^{-1}\right)=0 \quad \text { for } i=1, \ldots, m-1, \\
& T_{i} T_{i+1} T_{i}=T_{i+1} T_{i} T_{i+1} \quad \text { for } i=1, \ldots, m-2 \text {, } \\
& T_{i} T_{j}=T_{j} T_{i} \quad \text { for }|i-j| \geq 2 .
\end{aligned}
$$

If $w \in \mathfrak{S}_{m}$ is an element of the symmetric group on $m$ letters and $w=s_{i_{1}} s_{i_{2}} \ldots s_{i_{l}}$ is a reduced expression as a product of Coxeter generators, let $T_{w}:=T_{i_{1}} T_{i_{2}} \ldots T_{i_{l}}$. Then the set $\left\{T_{w}: w \in \mathfrak{S}_{m}\right\}$ is a basis of $\mathscr{H}_{m}$.

Note that $\mathscr{H}_{m}$ acts on $V^{\otimes m}$ since $\mathscr{H}_{m} \cong \mathfrak{B}_{m, 0}^{n}(q)$, the isomorphism given by $T_{i} \mapsto S_{i}$. 
Theorem 1.6 [Dipper and James 1989; Green 1996]. Let $\rho_{\text {ord }}: \mathbf{U} \rightarrow \operatorname{End}_{R}\left(V^{\otimes m}\right)$ be the representation of $\mathbf{U}$ on $V^{\otimes m}$. Then $\mathrm{im} \rho_{\mathrm{ord}}=\operatorname{End}_{\mathscr{H}_{m}}\left(V^{\otimes m}\right)$. This algebra is called the $q$-Schur algebra and denoted $S_{q}(n, m)$.

We will refer to $V^{\otimes m}$ as ordinary tensor space.

\section{Mixed tensor space as a submodule}

Recall that $\mathbf{U}^{\prime}$ is the subalgebra of $\mathbf{U}$ corresponding to the Lie algebra $\mathfrak{s l}_{n}$.

Theorem 2.1. If $m$ is a nonnegative integer, let $\rho_{\mathrm{ord}}: \mathbf{U} \rightarrow \operatorname{End}_{R}\left(V^{\otimes m}\right)$ be the representation of $\mathbf{U}$ on $V^{\otimes m}$. Then

$$
\rho_{\text {ord }}(\mathbf{U})=\rho_{\text {ord }}\left(\mathbf{U}^{\prime}\right) \text {. }
$$

Proof. Define the weight of $\boldsymbol{i} \in I(n, m)$ to be $\mathrm{wt}(\boldsymbol{i}):=\lambda=\left(\lambda_{1}, \ldots, \lambda_{n}\right)$ such that $\lambda_{i}$ is the number of entries in $i$ that are equal to $i$. If $\lambda=\left(\lambda_{1}, \ldots, \lambda_{n}\right)$ is a composition of $m$ into $n$ parts, i.e., $\lambda_{1}+\cdots+\lambda_{n}=m$, let $V_{\lambda}^{\otimes m}$ be the $R$-submodule of $V^{\otimes m}$ generated by all $v_{\mathbf{i}}$ with $\operatorname{wt}(\boldsymbol{i})=\lambda$. Then $V^{\otimes m}$ is the direct sum of all $V_{\lambda}^{\otimes m}$, where $\lambda$ runs through the set of compositions of $m$ into $n$ parts. Let $\varphi_{\lambda}$ be the projection onto $V_{\lambda}^{\otimes m}$. By [Green 1996], the restriction of $\rho_{\text {ord }}: \mathbf{U} \rightarrow S_{q}(n, m)$ to any subalgebra $\mathbf{U}^{\prime} \subseteq \mathbf{U}$ is surjective if the subalgebra $\mathbf{U}^{\prime}$ contains the divided powers $e_{i}^{(l)}$ and $f_{i}^{(l)}$ and preimages of the projections $\varphi_{\lambda}$.

Therefore, we define a partial order on the set of compositions of $m$ into $n$ parts by $\lambda \preceq \mu$ if and only if

$$
\left(\lambda_{1}-\lambda_{2}, \lambda_{2}-\lambda_{3}, \ldots, \lambda_{n-1}-\lambda_{n}\right) \leq\left(\mu_{1}-\mu_{2}, \mu_{2}-\mu_{3}, \ldots, \mu_{n-1}-\mu_{n}\right)
$$

in the lexicographical order. It suffices to show that for each composition $\lambda$, there exists an element $u \in \mathbf{U}^{\prime}$ such that $u v_{i}=0$ whenever $\operatorname{wt}(\boldsymbol{i}) \prec \lambda$ (i.e., $\operatorname{wt}(\boldsymbol{i}) \preceq \lambda$ and $\operatorname{wt}(\boldsymbol{i}) \neq \lambda)$ and $u v_{i}=v_{i}$ whenever $\operatorname{wt}(\boldsymbol{i})=\lambda$. In Theorem 4.5 of [Lusztig 1990], it is shown that certain elements

$$
\left[\begin{array}{c}
K_{i} ; c \\
t
\end{array}\right]:=\prod_{s=1}^{t} \frac{K_{i} q^{c-s+1}-K_{i}^{-1} q^{-c+s-1}}{q^{s}-q^{-s}}
$$

are elements of $\mathbf{U}^{\prime}$ for $i=1, \ldots, n-1, c \in \mathbb{Z}$ and $t \in \mathbb{N}$. Let

$$
u:=\prod_{i=1}^{n-1}\left[\begin{array}{c}
K_{i} ; m+1 \\
\lambda_{i}-\lambda_{i+1}+m+1
\end{array}\right],
$$

which is an element of $\mathbf{U}^{\prime}$ since $\lambda_{i}-\lambda_{i+1}+m+1>0$. Then $u$ has the desired properties.

The next lemma is motivated by [Dipper and Doty 2008, §6.3]. 
Lemma 2.2. There is a well defined $\mathbf{U}^{\prime}$-monomorphism $\kappa: V^{*} \rightarrow V^{\otimes n-1}$ given by

$$
\begin{aligned}
v_{i}^{*} & \mapsto(-q)^{i} \sum_{w \in \mathfrak{S}_{n-1}}(-q)^{l(w)} v_{(12 \ldots \hat{\imath} \ldots n) . w} \\
& =(-q)^{i} \sum_{w \in \mathfrak{S}_{n-1}}(-q)^{l(w)} v_{(12 \ldots \hat{\imath} \ldots . n)} T_{w}=(-q)^{i} v_{(12 \ldots \hat{\imath} \ldots n)} \sum_{w \in \mathfrak{S}_{n-1}}(-q)^{l(w)} T_{w},
\end{aligned}
$$

where $\hat{\imath}$ means leaving out $i$.

Proof. Clearly $\kappa$ is a monomorphism of $R$-modules, and $K_{i} v_{j}^{*}=q^{\delta_{i+1, j}-\delta_{i, j}} v_{j}^{*}$ and $K_{i} v_{(1 \ldots \hat{\jmath} \ldots n)}=q^{1-\delta_{i, j}} q^{\delta_{i+1, j}-1} v_{(1 \ldots \hat{j} \ldots n)}$ by definition. Thus, $\kappa$ commutes with $K_{i}$. Now $e_{i} v_{j}^{*}=-\delta_{i, j} q^{-1} v_{j+1}^{*}$. If $j \neq i, i+1$, then

$$
\begin{aligned}
e_{i} \kappa\left(v_{j}^{*}\right) & =(-q)^{j} e_{i} \sum_{w}(-q)^{l(w)} v_{(1 \ldots i i+1 \ldots \hat{j} \ldots n)} T_{w} \\
& =-(-q)^{j} \sum_{w}(-q)^{l(w)} v_{(1 \ldots i i \ldots \hat{\jmath} \ldots n)} T_{w}=0=\kappa\left(e_{i} v_{j}^{*}\right) .
\end{aligned}
$$

For $j=i$ (resp. $i+1)$, we get

$$
\begin{aligned}
e_{i} \kappa\left(v_{i+1}^{*}\right) & =(-q)^{i+1} \sum_{w}(-q)^{l(w)}\left(e_{i} v_{(1 \ldots \hat{i+1} \ldots n)}\right) T_{w}=0, \\
e_{i} \kappa\left(v_{i}^{*}\right) & =(-q)^{i} \sum_{w}(-q)^{l(w)}\left(e_{i} v_{(1 \ldots \hat{i} i+1 \ldots n)}\right) T_{w} \\
& =(-q)^{i} \sum_{w}(-q)^{l(w)} v_{(1 \ldots i \widehat{i+1} \ldots n)} T_{w}=-q^{-1} \kappa\left(v_{i+1}^{*}\right) .
\end{aligned}
$$

Furthermore, for $l \geq 2$ we clearly have $e_{i}^{(l)} v_{j}^{*}=0$ and $e_{i}^{(l)} \kappa\left(v_{j}^{*}\right)=0$. The argument for $f_{i}$ works similarly.

Lemma 2.2 enables us to consider the mixed tensor space $V^{\otimes r} \otimes V^{* \otimes s}$ as a $\mathbf{U}^{\prime}$-submodule $T^{r, s}$ of $V^{\otimes r+(n-1) s}$ via an embedding that we will also denote $\kappa$. Thus, $\mathfrak{B}_{r, s}^{n}(q)$ acts on $T^{r, s}$.

If we restrict the action of an element of $\mathbf{U}^{\prime}$ on $V^{\otimes r+(n-1) s}$ or equivalently of the $q$-Schur algebra $S_{q}(n, r+(n-1) s)$ to $T^{r, s}$, then we get an element of $\operatorname{End}_{R}\left(T^{r, s}\right)$. Since the actions of $\mathbf{U}^{\prime}$ and $\mathfrak{B}_{r, s}^{n}(q)$ commute, this is also an element of $\operatorname{End}_{\mathfrak{B}_{r, s}^{n}(q)}\left(T^{r, s}\right)$. Let $S_{q}(n ; r, s):=\operatorname{End}_{\mathfrak{B}_{r, s}^{n}(q)}\left(V^{\otimes r} \otimes V^{* \otimes s}\right)$; thus, we have an algebra homomorphism $\pi: S_{q}(n, r+(n-1) s) \rightarrow S_{q}(n ; r, s)$ by restriction of the action to $T^{r, s} \cong V^{\otimes r} \otimes V^{* \otimes s}$. Our aim is to show that $\pi$ is surjective, for then each element of $\operatorname{End}_{\mathfrak{B}_{r, s}^{n}(q)}\left(V^{\otimes r} \otimes V^{* \otimes s}\right)$ is given by the action of an element of $\mathbf{U}^{\prime}$.

Lemma 2.3. Let $M$ be a free $R$-module with basis $\mathscr{B}=\left\{b_{1}, \ldots, b_{l}\right\}$ and $U$ a submodule of $M$ given by a set of linear equations on the coefficients with respect to the basis $\Re$, i.e., $a_{i j} \in R$ such that $U=\left\{\sum c_{i} b_{i} \in M: \sum_{j} a_{i j} c_{j}=0\right.$ for all $\left.i\right\}$ 
exist. Let $\left\{b_{1}^{*}, \ldots, b_{l}^{*}\right\}$ be the basis of $M^{*}=\operatorname{Hom}_{R}(M, R)$ dual to $\mathscr{B}$, and let $X$ be the submodule generated by all $\sum_{j} a_{i j} b_{j}^{*}$. Then $U \cong\left(M^{*} / X\right)^{*}$.

Proof. We have that $\left(M^{*} / X\right)^{*}$ is isomorphic to the submodule of $M^{* *}$ given by linear forms on $M^{*}$ that vanish on $X$. Via the natural isomorphism $M^{* *} \cong M$, this is isomorphic to the set of elements of $M$ that are annihilated by $X$. An element $m=\sum_{k} c_{k} b_{k}$ is annihilated by $X$ if and only if $0=\sum_{j, k} a_{i j} b_{j}^{*}\left(c_{k} b_{k}\right)=\sum_{k} a_{i k} c_{k}$ for all $i$, and this is true if and only if $m \in U$.

Note an element $\tilde{\varphi} \in\left(M^{*} / X\right)^{*}$ corresponds to the element $\varphi=\sum_{i} \tilde{\varphi}\left(b_{i}^{*}+X\right) b_{i}$ of $U$. In our case, $S_{q}(n, m)$ and $S_{q}(n ; r, s)$ are $R$-submodules of $R$-free algebras, namely $\operatorname{End}_{R}\left(V^{\otimes m}\right)$ and $\operatorname{End}_{R}\left(V^{\otimes r} \otimes V^{* \otimes s}\right)$ respectively, given by a set of linear equations, which we will determine more precisely in Sections 3 and 4.

Definition 2.4. Let $M:=\operatorname{End}_{R}\left(V^{\otimes m}\right)$ and $U:=S_{q}(n, m)$. Then $U$ is defined as the algebra of endomorphisms commuting with a certain set of endomorphisms and thus is given by a system of linear equations on the coefficients. Let $A_{q}(n, m):=M^{*} / X$ as in Lemma 2.3. Similarly, let $A_{q}(n ; r, s):=M^{*} / X$ with $M:=\operatorname{End}_{R}\left(V^{\otimes r} \otimes V^{* \otimes s}\right)$ and $U:=S_{q}(n ; r, s)$.

By Lemma 2.3, $A_{q}(n, m)^{*}=S_{q}(n, m)$ and $A_{q}(n ; r, s)^{*}=S_{q}(n ; r, s)$. We will proceed as follows. We will take $m=r+(n-1) s$ and define an $R$-homomorphism $\iota: A_{q}(n ; r, s) \rightarrow A_{q}(n, r+(n-1) s)$ so that $\iota^{*}=\pi: S_{q}(n, r+(n-1) s) \rightarrow S_{q}(n ; r, s)$. Then we will define an $R$-homomorphism $\phi: A_{q}(n, r+(n-1) s) \rightarrow A_{q}(n ; r, s)$ such that $\phi \circ \iota=\mathrm{id}_{A_{q}(n ; r, s)}$ by giving suitable bases for $A_{q}(n, r+(n-1) s)$ and $A_{q}(n ; r, s)$. Dualizing this equation, we get $\pi \circ \phi^{*}=\iota^{*} \circ \phi^{*}=\mathrm{id}_{S_{q}(n ; r, s)}$, and this shows that $\pi$ is surjective. Actually, $A_{q}(n, r+(n-1) s)$ and $A_{q}(n ; r, s)$ are coalgebras, and $\iota$ is a morphism of coalgebras, but we do not need this for our results.

\section{3. $A_{q}(n, m)$}

The description of $A_{q}(n, m)$ is well known; see, e.g., [Dipper and Donkin 1991]. Let $A_{q}(n)$ be the free $R$-algebra on generators $x_{i j}(1 \leq i, j \leq n)$ subject to the relations

$$
\begin{aligned}
x_{i k} x_{j k} & =q x_{j k} x_{i k} & & \text { if } i<j, \\
x_{k i} x_{k j} & =q x_{k j} x_{k i} & & \text { if } i<j, \\
x_{i j} x_{k l} & =x_{k l} x_{i j} & & \text { if } i<k \text { and } j>l, \\
x_{i j} x_{k l} & =x_{k l} x_{i j}+\left(q-q^{-1}\right) x_{i l} x_{k j} & & \text { if } i<k \text { and } j<l .
\end{aligned}
$$

Note that these relations define the commutative algebra in $n^{2}$ commuting indeterminates $x_{i j}$ in case $q=1$. The free algebra on the generators $x_{i j}$ is obviously graded (with all generators in degree 1), and since the relations are homogeneous, this induces a grading on $A_{q}(n)$. Then we have the following lemma: 
Lemma 3.1 [Dipper and Donkin 1991]. $A_{q}(n, m)$ is the $R$-submodule of $A_{q}(n)$ of elements of homogeneous degree $m$.

Proof. Since our relations of the Hecke algebra differ from those in [Dipper and Donkin 1991] $\left(\left(T_{i}-q\right)\left(T_{i}+1\right)=0\right.$ is replaced by $\left.\left(T_{i}+q\right)\left(T_{i}-q^{-1}\right)=0\right)$ and thus $A_{q}(n, m)$ differs as well, we include a proof here.

Suppose $\varphi$ is an endomorphism of $V^{\otimes m}$ commuting with the action of a generator $S_{i}$. For convenience, we assume that $m=2$ and $S=S_{1}$. Then $\varphi$ can be written as a linear combination of the basis elements $E_{(i j),(k l)}$ mapping $v_{k} \otimes v_{l}$ to $v_{i} \otimes v_{j}$ and all other basis elements to 0 . For the coefficient of $E_{(i j),(k l)}$, we write $c_{i k} c_{j l}$ so that $\varphi=\sum_{i, j, k, l} c_{i k} c_{j l} E_{(i j),(k l)}$. On the one hand, we have

$S\left(\varphi\left(v_{k} \otimes v_{l}\right)\right)$

$=S\left(\sum_{i, j} c_{i k} c_{j l} v_{i} \otimes v_{j}\right)$

$=\sum_{i<j} c_{i k} c_{j l} v_{j} \otimes v_{i}+q^{-1} \sum_{i} c_{i k} c_{i l} v_{i} \otimes v_{i}+\sum_{i>j} c_{i k} c_{j l}\left(v_{j} \otimes v_{i}+\left(q^{-1}-q\right) v_{i} \otimes v_{j}\right)$

$=\sum_{i \neq j} c_{i k} c_{j l} v_{j} \otimes v_{i}+q^{-1} \sum_{i} c_{i k} c_{i l} v_{i} \otimes v_{i}+\left(q^{-1}-q\right) \sum_{i<j} c_{j k} c_{i l} v_{j} \otimes v_{i}$.

Now, suppose that $k>l$. Then

$$
\begin{aligned}
\varphi\left(S\left(v_{k} \otimes v_{l}\right)\right) & =\varphi\left(v_{l} \otimes v_{k}+\left(q^{-1}-q\right) v_{k} \otimes v_{l}\right) \\
& =\sum_{i, j}\left(c_{j l} c_{i k}+\left(q^{-1}-q\right) c_{j k} c_{i l}\right) v_{j} \otimes v_{i} .
\end{aligned}
$$

Similar formulas hold for $k=l$ and $k<l$. Comparing coefficients leads to the relations given above.

$A_{q}(n, m)$ has a basis consisting of monomials, but it will turn out to be more convenient for our purposes to work with a basis of standard bideterminants; see [Huang and Zhang 1993]. In that reference, the supersymmetric quantum letterplace algebra for $L^{-}=P^{-}=\{1, \ldots, n\}$ and $L^{+}=P^{+}=\varnothing$ is isomorphic to $A_{q^{-1}}(n) \cong$ $A_{q}(n)^{\mathrm{opp}}$, and we will adjust the results to our situation.

A partition $\lambda$ of $m$ is a sequence $\lambda=\left(\lambda_{1}, \lambda_{2}, \ldots, \lambda_{k}\right)$ of nonnegative integers such that $\lambda_{1} \geq \lambda_{2} \geq \cdots \geq \lambda_{k}$ and $\sum_{i=1}^{k} \lambda_{i}=m$. Denote the set of partitions of $m$ by $\Lambda^{+}(m)$. The Young diagram $[\lambda]$ of a partition $\lambda$ is $\left\{(i, j) \in \mathbb{N} \times \mathbb{N}: 1 \leq i \leq k, 1 \leq j \leq \lambda_{i}\right\}$. It can be represented by an array of boxes: $\lambda_{1}$ boxes in the first row, $\lambda_{2}$ boxes in the second row, etc.

A $\lambda$-tableau $\mathrm{t}$ is a map $f:[\lambda] \rightarrow\{1, \ldots, n\}$. A tableau can be represented by writing the entry $f(i, j)$ into the $(i, j)$ th box. A tableau $\mathfrak{t}$ is called standard if the entries in each row are strictly increasing from left to right and the entries in each 
column are nondecreasing downward. In the literature, this property is also called semistandard, and the role of rows and columns may be interchanged. Note that if $\mathfrak{t}$ is a standard $\lambda$-tableau, then $\lambda_{1} \leq n$. A pair $\left[\mathfrak{t}, \mathfrak{t}^{\prime}\right]$ of $\lambda$-tableaux is called a bitableau. It is standard if both $\mathfrak{t}$ and $\mathfrak{t}^{\prime}$ are standard $\lambda$-tableaux.

Note that the next definition differs from the definition in [Huang and Zhang 1993] by a sign.

Definition 3.2. Let $i_{1}, \ldots, i_{k}, j_{1}, \ldots, j_{k} \in\{1, \ldots, n\}$. For $i_{1}<i_{2}<\cdots<i_{k}$, let the right quantum minor be defined by

$$
\left(i_{1} i_{2} \ldots i_{k} \mid j_{1} j_{2} \ldots j_{k}\right)_{r}:=\sum_{w \in \mathfrak{S}_{k}}(-q)^{l(w)} x_{i_{w 1} j_{1}} x_{i_{w 2} j_{2}} \ldots x_{i_{w k} j_{k}} .
$$

For arbitrary $i_{1}, \ldots, i_{k}$, the right quantum minor is then defined by the rule

$$
\left(i_{1} \ldots i_{l} i_{l+1} \ldots i_{k} \mid j_{1} j_{2} \ldots j_{k}\right)_{r}:=-q^{-1}\left(i_{1} \ldots i_{l-1} i_{l+1} i_{l} i_{l+2} \ldots i_{k} \mid j_{1} j_{2} \ldots j_{k}\right)_{r}
$$

for $i_{l}>i_{l+1}$. Similarly, let the left quantum minor be defined by

$$
\begin{aligned}
\left(i_{1} \ldots i_{k} \mid j_{1} \ldots j_{k}\right)_{l}: & =\sum_{w \in \mathfrak{S}_{k}}(-q)^{l(w)} x_{i_{1}, j_{w 1}} x_{i_{2} j_{w 2}} \ldots x_{i_{k} j_{w k}} \text { if } j_{1}<\ldots<j_{k}, \\
\left(i_{1} \ldots i_{k} \mid j_{1} \ldots j_{k}\right)_{l}: & =-q^{-1}\left(i_{1} \ldots i_{k} \mid j_{1} \ldots j_{l+1} j_{l} \ldots j_{k}\right)_{l} \text { if } j_{l}>j_{l+1} .
\end{aligned}
$$

Finally, let the quantum determinant be defined by

$$
\operatorname{det}_{q}:=(12 \ldots n \mid 12 \ldots n)_{r}=(12 \ldots n \mid 12 \ldots n)_{l} .
$$

If $\left[\mathfrak{t}, \mathfrak{t}^{\prime}\right]$ is a bitableau and $\mathfrak{t}_{1}, \mathfrak{t}_{2}, \ldots, \mathfrak{t}_{k}\left(\right.$ resp. $\left.\mathfrak{t}_{1}^{\prime}, \mathfrak{t}_{2}^{\prime}, \ldots, \mathfrak{t}_{k}^{\prime}\right)$ are the rows of $\mathfrak{t}\left(\right.$ resp. $\left.\mathfrak{t}^{\prime}\right)$, then let

$$
\left(\mathfrak{t} \mid \mathfrak{t}^{\prime}\right):=\left(\mathfrak{t}_{k} \mid \mathfrak{t}_{k}^{\prime}\right)_{r} \ldots\left(\mathfrak{t}_{2} \mid \mathfrak{t}_{2}^{\prime}\right)_{r}\left(\mathfrak{t}_{1} \mid \mathfrak{t}_{1}^{\prime}\right)_{r} .
$$

Then $\left(\mathfrak{t} \mid \mathfrak{t}^{\prime}\right)$ is called a bideterminant.

Remark 3.3. We note the following properties of quantum minors:

(1) $\left(i_{1} \ldots i_{k} \mid j_{1} \ldots j_{k}\right)_{r}=-q\left(i_{1} \ldots i_{k} \mid j_{1} \ldots j_{l+1} j_{l} \ldots j_{k}\right)_{r}$ for $j_{l}>j_{l+1}$, $\left(i_{1} \ldots i_{k} \mid j_{1} \ldots j_{k}\right)_{l}=-q\left(i_{1} \ldots i_{l+1} i_{l} \ldots i_{k} \mid j_{1} \ldots j_{k}\right)_{l}$ for $i_{l}>i_{l+1}$.

(2) If $i_{1}<i_{2}<\cdots<i_{k}$ and $j_{1}<j_{2}<\cdots<j_{k}$, then right and left quantum minors coincide, and we simply write $\left(i_{1} \ldots i_{k} \mid j_{1} \ldots j_{k}\right)$. This notation thus indicates that the sequences of numbers are increasing. In general, right and left quantum minors differ by a power of $-q$.

(3) If two $i_{l}$ s or $j_{l}$ s coincide, then the quantum minors vanish.

(4) The quantum determinant $\operatorname{det}_{q}$ is an element of the center of $A_{q}(n)$. 
Definition 3.4. Let the content of a monomial $x_{i_{1} j_{1}} \ldots x_{i_{m} j_{m}}$ be defined as the tuple $(\alpha, \beta)=\left(\left(\alpha_{1}, \ldots, \alpha_{n}\right),\left(\beta_{1}, \ldots, \beta_{n}\right)\right)$, where $\alpha_{i}$ is the number of indices $i_{t}$ such that $i_{t}=i$ and $\beta_{j}$ is the number of indices $j_{t}$ such that $j_{t}=j$. Note that $\sum \alpha_{i}=\sum \beta_{j}=m$ for each monomial of homogeneous degree $m$. For such a tuple $(\alpha, \beta)$, let $P(\alpha, \beta)$ be the subspace of $A_{q}(n, m)$ generated by the monomials of content $(\alpha, \beta)$. Furthermore, let the content of a bitableau $\left[\mathfrak{t}, \mathfrak{t}^{\prime}\right]$ be defined similarly as the tuple $(\alpha, \beta)$ such that $\alpha_{i}$ is the number of entries in $\mathfrak{t}$ equal to $i$ and $\beta_{j}$ is the number of entries in $\mathfrak{t}^{\prime}$ equal to $j$.

Theorem 3.5 [Huang and Zhang 1993]. The bideterminants $\left(\mathfrak{t} \mid \mathfrak{t}^{\prime}\right)$ of the standard $\lambda$-tableaux with $\lambda$ a partition of $m$ form a basis of $A_{q}(n, m)$ such that the bideterminants of standard $\lambda$-tableaux of content $(\alpha, \beta)$ form a basis of $P(\alpha, \beta)$.

The proof in [Huang and Zhang 1993] works over a field, but the arguments are valid if the field is replaced by a commutative ring with 1 . The reversed order of the minors is due to the opposite algebra. Note that for $i_{1}<i_{2}<\cdots<i_{k}$ and $j_{1}<j_{2}<\cdots<j_{k}$, we have

$$
q^{k(k-1) / 2}\left(i_{1} i_{2} \ldots i_{k} \mid j_{1} j_{2} \ldots j_{k}\right)_{r}=\sum_{w \in \mathfrak{S}_{k}}(-q)^{-l(w)} x_{i_{w k} j_{1}} x_{i_{w(k-1)} j_{2}} \ldots x_{i_{w 1} j_{k}},
$$

which is a quantum minor of $A_{q^{-1}}(n)^{\mathrm{opp}}$.

Lemma 3.6 (Laplace's expansion [Huang and Zhang 1993]).

(1) For $j_{1}<j_{2}<\cdots<j_{l}<j_{l+1}<\cdots<j_{k}$, we have

$\left(i_{1} i_{2} \ldots i_{k} \mid j_{1} j_{2} \ldots j_{k}\right)_{l}$

$$
=\sum_{w}(-q)^{l(w)}\left(i_{1} \ldots i_{l} \mid j_{w 1} \ldots j_{w l}\right)_{l}\left(i_{l+1} \ldots i_{k} \mid j_{w(l+1)} \ldots j_{w k}\right)_{l},
$$

where the summation is over all $w \in \mathfrak{S}_{k}$ such that $w 1<w 2<\cdots<w l$ and $w(l+1)<w(l+2)<\cdots<w k$.

(2) For $i_{1}<i_{2}<\cdots<i_{k}$, we have

$$
\begin{aligned}
& \left(i_{1} i_{2} \ldots i_{k} \mid j_{1} j_{2} \ldots j_{k}\right)_{r} \\
& \qquad \sum_{w}(-q)^{l(w)}\left(i_{w 1} \ldots i_{w l} \mid j_{1} \ldots j_{l}\right)_{r}\left(i_{w(l+1)} \ldots i_{w k} \mid j_{l+1} \ldots j_{k}\right)_{r},
\end{aligned}
$$

the summation again over all $w \in \mathfrak{S}_{k}$, such that $w 1<w 2<\cdots<w l$ and $w(l+1)<w(l+2)<\cdots<w k$.

\section{4. $A_{q}(n ; r, s)$}

A basis of $\operatorname{End}_{R}\left(V^{\otimes r} \otimes V^{* \otimes s}\right)$ is given by matrix units $E_{\boldsymbol{i}|\boldsymbol{j} k| \boldsymbol{l}}$ such that $E_{\boldsymbol{i}|\boldsymbol{j} \boldsymbol{k}| \boldsymbol{l}} v_{\mathbf{s} \mid \boldsymbol{t}}=$ $\delta_{\boldsymbol{k}|\boldsymbol{l}, \boldsymbol{s}| \mathbf{t}} v_{\boldsymbol{i} \mid \boldsymbol{j}}$. Suppose $\varphi:=\sum_{\mathbf{i}, \boldsymbol{j}, \boldsymbol{k}, \boldsymbol{l}} c_{\boldsymbol{i}|\boldsymbol{j} \mathbf{k}| \boldsymbol{l}} E_{\boldsymbol{i}|\boldsymbol{j} \boldsymbol{k}| \boldsymbol{l}} \in \operatorname{End}_{R}\left(V^{\otimes r} \otimes V^{* \otimes s}\right)$ commutes 
with the action of $\mathfrak{B}_{r, s}^{n}(q)$ or equivalently with a set of generators of $\mathfrak{B}_{r, s}^{n}(q)$. Since coefficient spaces are multiplicative, we can write

$$
c_{i_{1} k_{1}} c_{i_{2} k_{2}} \cdots c_{i_{r} k_{r}} c_{j_{1} l_{1}}^{*} c_{j_{2} l_{2}}^{*} \cdots c_{j_{s} l_{s}}^{*}
$$

for the coefficient $c_{i \mid j} k \mid l$. It is easy to see from the description of $A_{q}(n, m)$ that $\varphi$ commutes with the generators without nonpropagating edges if and only if the $c_{i j}$ satisfy the relations of $A_{q}(n)$ and the $c_{i j}^{*}$ satisfy the relations of $A_{q^{-1}}(n) \cong A_{q}(n)^{\mathrm{opp}}$.

Now suppose that $\varphi$ in addition commutes with the action of the generator

$$
e=\downarrow \cdots \downarrow \stackrel{\leftrightarrow}{\leftrightarrow} \uparrow \uparrow \text {. }
$$

We assume $\varphi=\sum_{i, j, k, l=1}^{n} c_{i k} c_{j l}^{*} E_{i|j k| l}$ and that $r=s=1$ (the general case being similar). Let $v=v_{i} \otimes v_{j}^{*}$ be a basis element of $V \otimes V^{*}$. We have (the indices in the sums always run from 1 to $n$ )

$$
\begin{aligned}
& \varphi(v) e=\sum_{s, t} c_{s i} c_{t j}^{*}\left(v_{s} \otimes v_{t}^{*}\right) e=\sum_{s, k} q^{2 s-n-1} c_{s i} c_{s j}^{*}\left(v_{k} \otimes v_{k}^{*}\right), \\
& \varphi(v e)=\delta_{i j} q^{2 i-n-1} \sum_{k} \varphi\left(v_{k} \otimes v_{k}^{*}\right)=\delta_{i j} q^{2 i-n-1} \sum_{k, s, t} c_{s k} c_{t k}^{*} v_{s} \otimes v_{t}^{*} .
\end{aligned}
$$

Comparing coefficients, we get the following conditions:

$$
\begin{gathered}
\sum_{k=1}^{n} c_{i k} c_{j k}^{*}=0 \quad \text { for } i \neq j, \\
\sum_{k=1}^{n} q^{2 k} c_{k i} c_{k j}^{*}=0 \quad \text { for } i \neq j, \\
\sum_{k=1}^{n} q^{2 k-2 i} c_{k i} c_{k i}^{*}=\sum_{k=1}^{n} c_{j k} c_{j k}^{*} .
\end{gathered}
$$

This, combined with Lemma 2.3, shows the following:

Lemma 4.1. We have

$$
A_{q}(n ; r, s) \cong\left(F(n, r) \otimes_{R} F_{*}(n, s)\right) / Y,
$$

where $F(n, r)$ (resp. $\left.F_{*}(n, s)\right)$ is the $R$-submodule of the free algebra on generators $x_{i j}$ (resp. $x_{i j}^{*}$ ) generated by monomials of degree $r$ (resp. $s$ ) and $Y$ is the $R$-submodule of $F(n, r) \otimes_{R} F_{*}(n, s)$ generated by elements of the form $h_{1} h_{2} h_{3}$, where $h_{2}$ is one of the elements

$$
\begin{array}{ll}
x_{i k} x_{j k}-q x_{j k} x_{i k} & \text { for } i<j, \\
x_{k i} x_{k j}-q x_{k j} x_{k i} & \text { for } i<j, \\
x_{i j} x_{k l}-x_{k l} x_{i j} & \text { for } i<k, j>l,
\end{array}
$$




$$
\begin{array}{ll}
x_{i j} x_{k l}-x_{k l} x_{i j}-\left(q-q^{-1}\right) x_{i l} x_{k j} & \text { for } i<k, j<l, \\
x_{i k}^{*} x_{j k}^{*}-q^{-1} x_{j k}^{*} x_{i k}^{*} & \text { for } i<j, \\
x_{k i}^{*} x_{k j}^{*}-q^{-1} x_{k j}^{*} x_{k i}^{*} & \text { for } i<j, \\
x_{i j}^{*} x_{k l}^{*}-x_{k l}^{*} x_{i j}^{*} & \text { for } i<k, j>l, \\
x_{i j}^{*} x_{k l}^{*}-x_{k l}^{*} x_{i j}^{*}+\left(q-q^{-1}\right) x_{i l}^{*} x_{k j}^{*} & \text { for } i<k, j<l, \\
\sum_{k=1}^{n} x_{i k} x_{j k}^{*} & \text { for } i \neq j, \\
\sum_{k=1}^{n} q^{2 k} x_{k i} x_{k j}^{*} & \text { for } i \neq j, \\
\sum_{k=1}^{n} q^{2 k-2 i} x_{k i} x_{k i}^{*}-\sum_{k=1}^{n} x_{j k} x_{j k}^{*} &
\end{array}
$$

and $h_{1}$ and $h_{3}$ are monomials of appropriate degree.

Remark 4.2. The map given by $x_{i k} \mapsto q^{2 k-2 i} x_{k i}$ and $x_{i k}^{*} \mapsto x_{k i}^{*}$ induces an $R$-linear automorphism of $A_{q}(n ; r, s)$.

Bideterminants can also be formed using the variables $x_{i j}^{*}$. In this case, let

$$
\left(\mathfrak{t} \mid \mathfrak{t}^{\prime}\right)^{*}:=\left(\mathfrak{t}_{1} \mid \mathfrak{t}_{1}^{\prime}\right)_{r}^{*}\left(\mathfrak{t}_{2} \mid \mathfrak{t}_{2}^{\prime}\right)_{r}^{*} \cdots\left(\mathfrak{t}_{k} \mid \mathfrak{t}_{k}^{\prime}\right)_{r}^{*},
$$

where the quantum minors $\left(i_{1} \ldots i_{k} \mid j_{1} \ldots j_{k}\right)_{r / l}^{*}$ are defined as above with $q$ replaced by $q^{-1}$.

\section{The map $\iota: A_{q}(n ; r, s) \rightarrow A_{q}(n, r+(n-1) s)$}

For any $1 \leq i, j \leq n$, let $\iota\left(x_{i j}\right):=x_{i j}$ and

$$
\iota\left(x_{i j}^{*}\right):=(-q)^{j-i}(12 \ldots \hat{\imath} \ldots n \mid 12 \ldots \hat{\jmath} \ldots n) \in A_{q}(n, n-1) ;
$$

then there is a unique $R$-linear map

$$
\iota: F(n, r) \otimes_{R} F_{*}(n, s) \rightarrow A_{q}(n, r+(n-1) s)
$$

such that $\iota\left(x_{i_{1} j_{1}} \cdots x_{i_{r} j_{r}} x_{k_{1} l_{1}}^{*} \cdots x_{k_{s} l_{s}}^{*}\right)=\iota\left(x_{i_{1} j_{1}}\right) \cdots \iota\left(x_{i_{r} j_{r}}\right) \iota\left(x_{k_{1} l_{1}}^{*}\right) \cdots \iota\left(x_{k_{s} l_{s}}^{*}\right)$.

Lemma 5.1. The kernel of $\iota$ contains $Y$, and thus, $\iota$ induces an R-linear map

$$
A_{q}(n ; r, s) \rightarrow A_{q}(n, r+(n-1) s),
$$

which we will then also denote $\iota$. 
Proof. We have to show that the generators of $Y$ lie in the kernel of $\iota$. Generators of $Y$ involving the elements (4.1.1)-(4.1.4) are obviously in the kernel of $\iota$. Theorem 7.3 of [Goodearl 2006] shows that generators involving elements (4.1.5)-(4.1.8) are also in the kernel. Laplace's expansion shows that

$$
\begin{aligned}
\iota\left(\sum_{k=1}^{n} x_{i k} x_{j k}^{*}\right) & =\sum_{k=1}^{n}(-q)^{(k-1)-(j-1)} x_{i k} \cdot(1 \ldots \hat{\jmath} \ldots n \mid 1 \ldots \hat{k} \ldots n)_{l} \\
& =(-q)^{1-j}(i 1 \ldots \hat{\jmath} \ldots n \mid 1 \ldots n)_{l}=\delta_{i, j} \cdot \operatorname{det}_{q} \\
\iota\left(\sum_{k=1}^{n} q^{2 k-2 i} x_{k i} x_{k j}^{*}\right) & =q^{-2 i+j+1} \sum_{k=1}^{n}(-q)^{k-1} x_{k i} \cdot(1 \ldots \hat{k} \ldots n \mid 1 \ldots \hat{\jmath} \ldots n)_{r} \\
& =(-q)^{j-2 i+1}(1 \ldots n \mid i 1 \ldots \hat{\jmath} \ldots n)_{r}=\delta_{i, j} \cdot \operatorname{det}_{q}
\end{aligned}
$$

thus, the generators involving the elements (4.1.9)-(4.1.11) are in the kernel of $\iota$.

Now, we have maps

$\iota^{*}: A_{q}(n, r+(n-1) s)^{*} \rightarrow A_{q}(n ; r, s)^{*} \quad$ and $\quad \pi: S_{q}(n, r+(n-1) s) \rightarrow S_{q}(n ; r, s)$.

By definition, $A_{q}(n, r+(n-1) s)^{*} \cong S_{q}(n, r+(n-1) s)$ and $A_{q}(n ; r, s)^{*} \cong S_{q}(n ; r, s)$.

Lemma 5.2. Under the identifications above, we have $\iota^{*}=\pi$.

Proof. We will write

$$
\begin{aligned}
x_{i_{1} \ldots i_{l} j_{1} \ldots j_{l}} & =x_{i_{1}, j_{1}} \cdots x_{i_{l}, j_{l}}, \\
x_{i_{l} \ldots i_{1} \mid l_{1} \ldots l_{m}} j_{l} \ldots j_{1} \mid k_{1} \ldots k_{m} & =x_{i_{l}, j_{l}} \cdots x_{i_{1}, j_{1}} x_{l_{1}, k_{1}}^{*} \cdots x_{l_{m}, k_{m}}^{*} .
\end{aligned}
$$

Suppose that $\tilde{\varphi} \in A_{q}(n, r+(n-1) s)^{*}$. Then

$$
\varphi=\sum_{\boldsymbol{i}, \boldsymbol{j} \in \mathrm{I}(n, r+(n-1) s)} \tilde{\varphi}\left(x_{\mathbf{i} j}\right) E_{\boldsymbol{i j}}
$$

is the corresponding element of $S_{q}(n, r+(n-1) s)$. Since $\iota^{*}(\tilde{\varphi})=\tilde{\varphi} \circ \iota$, we have

$$
\iota^{*}(\varphi)=\sum_{\boldsymbol{i}, \boldsymbol{j}, \boldsymbol{k}, \boldsymbol{l}} \tilde{\varphi} \circ \iota\left(x_{\boldsymbol{i}|\boldsymbol{j} \boldsymbol{k}| \boldsymbol{l}}\right) E_{\boldsymbol{i}|\boldsymbol{j} \boldsymbol{k}| \boldsymbol{l}}
$$

In other words, the coefficient of $E_{\boldsymbol{i}|\boldsymbol{j} \boldsymbol{k}| \boldsymbol{l}}$ in $\iota^{*}(\varphi)$ can be computed by substituting each $x_{\mathbf{s} t}$ in $\iota\left(x_{i|j k| l}\right)$ by $\tilde{\varphi}\left(x_{\mathrm{s} t}\right)$. On the other hand, to compute the coefficient of $E_{\boldsymbol{i}|\boldsymbol{j} \boldsymbol{k}| \boldsymbol{l}}$ in $\pi(\varphi)$, one has to consider the action of $\varphi$ on a basis element $v=\kappa\left(v_{\boldsymbol{k} \mid \boldsymbol{l}}\right)$ of $T^{r, s}$. For a multi-index $\boldsymbol{l} \in \mathrm{I}(n, s)$, let $\boldsymbol{l}^{*} \in \mathrm{I}(n,(n-1) s)$ be defined by

$$
l^{*}:=\left(1 \ldots \widehat{l_{1}} \ldots n 1 \ldots \widehat{l_{2}} \ldots n \ldots 1 \ldots \widehat{l_{s}} \ldots n\right) .
$$


Then

$$
v=\kappa\left(v_{\boldsymbol{k} \mid l}\right)=(-q)^{l_{1}+l_{2}+\cdots+l_{s}} \sum_{w \in \mathfrak{S}_{n-1}^{\times s}}(-q)^{l(w)} v_{\boldsymbol{k}} \otimes\left(v_{l^{*}} T_{w}\right),
$$

and thus, we have

$$
\begin{aligned}
\varphi(v) & =(-q)^{\sum l_{k}} \sum_{\boldsymbol{s}, \boldsymbol{t}, w}(-q)^{l(w)} \tilde{\varphi}\left(x_{\boldsymbol{s} t}\right) E_{\boldsymbol{s} \boldsymbol{t}}\left(v_{\boldsymbol{k}} \otimes\left(v_{\boldsymbol{l}^{*}} T_{w}\right)\right) \\
& =\sum_{\boldsymbol{s}, w}(-q)^{l(w)+\sum l_{k}} \tilde{\varphi}\left(x_{\boldsymbol{s}} \boldsymbol{k} \boldsymbol{l}^{*} * w\right) v_{\boldsymbol{s}} .
\end{aligned}
$$

Since $\varphi$ leaves $T^{r, s}$ invariant, $\varphi(v)$ is a linear combination of the basis elements $\kappa\left(v_{i \mid j}\right)$ of $T^{r, s}$. Distinct $\kappa\left(v_{i \mid j}\right)$ involve distinct basis vectors of $V^{\otimes r+(n-1) s}$. Thus, if

$$
\varphi(v)=\sum_{i \mid \boldsymbol{j}} \lambda_{\boldsymbol{i} \mid \boldsymbol{j}} \kappa\left(v_{\boldsymbol{i} \mid \boldsymbol{j}}\right)=\sum_{\boldsymbol{i} \mid \boldsymbol{j}, w} \lambda_{\boldsymbol{i} \mid \boldsymbol{j}}(-q)^{l(w)+j_{1}+\cdots+j_{s}} v_{\boldsymbol{i} \boldsymbol{j}^{*} . w},
$$

then $(-q)^{\sum j_{k}} \lambda_{\boldsymbol{i} \mid \boldsymbol{j}}$ is equal to the coefficient of $\boldsymbol{v}_{\boldsymbol{i} \boldsymbol{j}^{*}}$ when $\varphi(v)$ is written as a linear combination of basis vectors of $V^{\otimes r+(n-1) s}$. The coefficient of $v_{i j^{*}}$ in $\varphi(v)$ is, by the formula above,

$$
(-q)^{\sum l_{k}} \sum_{w}(-q)^{l(w)} \tilde{\varphi}\left(x_{i j^{*}} \boldsymbol{k l} \boldsymbol{l}^{*} w\right) .
$$

Thus,

$$
\lambda_{\boldsymbol{i} \mid \boldsymbol{j}}=(-q)^{\sum l_{k}-j_{k}} \sum_{w}(-q)^{l(w)} \tilde{\varphi}\left(x_{\boldsymbol{i} \boldsymbol{j}^{*} \boldsymbol{k} \boldsymbol{l}^{*} w}\right)=\tilde{\varphi} \circ \iota\left(x_{\boldsymbol{i}|\boldsymbol{j} \boldsymbol{k}| l}\right) .
$$

But $\lambda_{\boldsymbol{i} \mid \boldsymbol{j}}$ is also the coefficient of $E_{\boldsymbol{i}|\boldsymbol{j} \boldsymbol{k}| \boldsymbol{l}}$ in $\pi(\varphi)$, which shows the result.

Theorem 5.3 (Jacobi's ratio theorem). Suppose $n \geq l \geq 0$ and $i_{1}<i_{2}<\cdots<i_{l}$ and $j_{1}<j_{2}<\cdots<j_{l}$. Let $i_{1}^{\prime}<i_{2}^{\prime}<\cdots<i_{n-l}^{\prime}$ and $j_{1}^{\prime}<j_{2}^{\prime}<\cdots<j_{n-l}^{\prime}$ be the unique numbers such that $\{1, \ldots, n\}=\left\{i_{1}, \ldots, i_{l}, i_{1}^{\prime}, \ldots, i_{n-l}^{\prime}\right\}=\left\{j_{1}, \ldots, j_{l}, j_{1}^{\prime}, \ldots, j_{n-l}^{\prime}\right\}$. Then

$$
\iota\left(\left(i_{1} \ldots i_{l} \mid j_{1} \ldots j_{l}\right)^{*}\right)=(-q)^{\sum_{t=1}^{l}\left(j_{t}-i_{t}\right)} \operatorname{det}_{q}^{l-1}\left(i_{1}^{\prime} \ldots i_{n-l}^{\prime} \mid j_{1}^{\prime} \ldots j_{n-l}^{\prime}\right) .
$$

Proof. We argue by induction on $l$. Note that for $l=0, \operatorname{det}_{q}^{l-1}=\operatorname{det}_{q}^{-1}$ is not an element of $A_{q}(n)$. However, $\left(i_{1}^{\prime} \ldots i_{n-l}^{\prime} \mid j_{1}^{\prime} \ldots j_{n-l}^{\prime}\right)$ turns out to be $\operatorname{det}_{q}$; thus, the right-hand side of the formula is $\operatorname{det}_{q}^{-1} \operatorname{det}_{q}=1=\iota(1)$. In this sense, the formula is valid for $l=0$.

For $l=1$, the theorem is true by the definition of $\iota\left(x_{i j}^{*}\right)$. Now assume the theorem is true for $l-1$. Apply Laplace's expansion and use induction to get $\iota\left(\left(i_{1} \ldots i_{l} \mid j_{1} \ldots j_{l}\right)^{*}\right)=\iota\left(\sum_{k=1}^{l}(-q)^{-(k-1)} x_{i_{k} j_{1}}^{*}\left(i_{1} \ldots \widehat{i_{k}} \ldots i_{l} \mid j_{2} \ldots \ldots j_{l}\right)^{*}\right)$ 


$$
\begin{aligned}
& =\sum_{k=1}^{l}(-q)^{1-k}(-q)^{j_{1}-i_{k}}\left(1 \ldots \widehat{i_{k}} \ldots n \mid 1 \ldots \widehat{j_{1}} \ldots n\right) \cdot(-q)^{\sum_{t \neq 1} j_{t}-\sum_{t \neq k} i_{t}} \operatorname{det}_{q}^{l-2} \\
& \quad \cdot\left(1 \ldots \widehat{i_{1}} \ldots \widehat{i_{2}} \ldots . \hat{i_{k-1}} \ldots \widehat{i_{k+1}} \ldots \hat{i_{l}} \ldots n \mid 1 \ldots \widehat{j_{2}} \ldots \widehat{j_{3}} \ldots . \widehat{j_{l}} \ldots n\right) .
\end{aligned}
$$

We claim that this is equal to

$$
\begin{array}{r}
(-q)^{\sum_{t=1}^{l}\left(j_{t}-i_{t}\right)} \operatorname{det}_{q}^{l-2} \sum_{w}(-q)^{l(w)+1-n}\left(w 1 w 2 \ldots w(n-1) \mid 1 \ldots \widehat{j_{1}} \ldots n\right) \\
\cdot\left(w n 1 \ldots \widehat{i_{1}} \ldots . \widehat{i_{l}} \ldots n \mid 1 \ldots \widehat{j_{2}} \ldots \ldots \widehat{j_{l}} \ldots n\right)_{l},
\end{array}
$$

where the summation is over all $w \in \mathfrak{S}_{n}$ such that $w 1<w 2<\cdots<w(n-1)$. If $w n$ is not one of the $i_{k} \mathrm{~s}$, then the summand in (5.3.1) vanishes since $w n$ appears twice in the row on the left side of the second minor. Thus, the summation is over all $w$ as above with $w n=i_{k}$ for some $k$. Note that $l(w)=n-i_{k}$ and

$$
\left(i_{k} 1 \ldots \widehat{i_{1}} \ldots . \hat{i_{l}} \ldots n \mid \mathfrak{t}\right)_{l}=(-q)^{i_{k}-k}\left(1 \ldots \widehat{i_{1}} \ldots \widehat{i_{k-1}} \ldots \hat{i_{k+1}} \ldots \widehat{i_{l}} \ldots n \mid \mathfrak{t}\right) ;
$$

the claim follows. Again apply Laplace's expansion to the second minor in (5.3.1) to get

$$
\begin{aligned}
& \left(w n 1 \ldots \widehat{i_{1}} \ldots . \widehat{i_{l}} \ldots n \mid 1 \ldots \widehat{j_{2}} \ldots \ldots \widehat{j_{l}} \ldots n\right)_{l} \\
& \quad=\sum_{v}(-q)^{l(v)} x_{w n v 1}\left(1 \ldots \widehat{i_{1}} \ldots . \hat{i_{l}} \ldots n \mid v 2 v 3 \ldots \widehat{v j_{2}} \ldots \ldots \widehat{v j_{l}} \ldots v n\right),
\end{aligned}
$$

the summation being over all $v \in \mathfrak{S}_{\left\{1, \ldots, \widehat{j}_{2}, \ldots, \widehat{j}_{l}, \ldots, n\right\}}$ with $v 2<v 3<\cdots<v n$. After substituting this term in (5.3.1), one can again apply Laplace's expansion to get that (5.3.1) is equal to

$$
\begin{aligned}
(-q)^{\sum\left(j_{t}-i_{t}\right)} \operatorname{det}_{q}^{l-2} \sum_{v}(-q)^{l(v)+1-n}\left(12 \ldots n \mid 1 \ldots \widehat{j_{1}} \ldots n v 1\right)_{r} \\
\cdot\left(1 \ldots \widehat{i_{1}} \ldots \ldots \widehat{i_{l}} \ldots n \mid v 2 v 3 \ldots \widehat{j_{2}} \ldots \ldots \widehat{v j_{l}} \ldots v n\right) .
\end{aligned}
$$

The only summand in (5.3.2) that does not vanish is the term for $v 1=j_{1}$ with $l(v)=j_{1}-1$. Thus, (5.3.2) is equal to

$$
\begin{array}{r}
(-q)^{\sum\left(j_{t}-i_{t}\right)} \operatorname{det}_{q}^{l-2}(-q)^{j_{1}-n}\left(12 \ldots n \mid 1 \ldots \widehat{j_{1}} \ldots n j_{1}\right)_{r} \cdot\left(i_{1}^{\prime} \ldots i_{n-l}^{\prime} \mid j_{1}^{\prime} \ldots j_{n-l}^{\prime}\right) \\
=(-q)^{\sum_{t=1}^{l}\left(j_{t}-i_{t}\right)} \operatorname{det}_{q}^{l-1}\left(i_{1}^{\prime} \ldots i_{n-l}^{\prime} l j_{1}^{\prime} \ldots j_{n-l}^{\prime}\right) .
\end{array}
$$

\section{A basis for $A_{q}(n ; r, s)$}

Theorem 5.3 enables us to construct elements of $A_{q}(n ; r, s)$ that are mapped to standard bideterminants under $\iota$. First, we will introduce the notion of rational tableaux although we will slightly differ from the definition of rational tableaux in [Stembridge 1987]. Recall that $\Lambda^{+}(k)$ is the set of partitions of $k$. 
Definition 6.1. Fix $0 \leq k \leq \min (r, s)$. Let $\rho \in \Lambda^{+}(r-k)$ and $\sigma \in \Lambda^{+}(s-k)$ with $\rho_{1}+\sigma_{1} \leq n$. A rational $(\rho, \sigma)$-tableau is a pair $(\mathfrak{r}, \mathfrak{s})$ with $\mathfrak{r}$ a $\rho$-tableau and $\mathfrak{s}$ a $\sigma$-tableau.

Let $\operatorname{first}_{i}(\mathfrak{r}, \mathfrak{s})$ be the number of entries of the first row of $\mathfrak{r}$, which are at most $i$, plus the number of entries of the first row of $\mathfrak{s}$, which are at most $i$. A rational tableau is called standard if $\mathfrak{r}$ and $\mathfrak{s}$ are standard tableaux and the following condition holds:

$$
\text { first }_{i}(\mathfrak{r}, \mathfrak{s}) \leq i \quad \text { for all } i=1, \ldots, n .
$$

A pair $\left[(\mathfrak{r}, \mathfrak{s}),\left(\mathfrak{r}^{\prime}, \mathfrak{s}^{\prime}\right)\right]$ of rational $(\rho, \sigma)$-tableaux is called a rational bitableau, and it is called a standard rational bitableau if both $(\mathfrak{r}, \mathfrak{s})$ and $\left(\mathfrak{r}^{\prime}, \mathfrak{s}^{\prime}\right)$ are standard rational tableaux.

Remark 6.2. In [Stembridge 1987], condition (6.1.1) is already part of the definition of rational tableaux. The condition $\rho_{1}+\sigma_{1} \leq n$ is equivalent to condition (6.1.1) for $i=n$. The reason for the difference will be apparent in the next lemma's proof.

Lemma 6.3. There is a bijection between the set consisting of all standard rational $(\rho, \sigma)$-tableaux for $\rho \in \Lambda^{+}(r-k)$ and $\sigma \in \Lambda^{+}(s-k)$ as $k$ runs from 0 to $\min (r, s)$ and the set of all standard $\lambda$-tableaux for $\lambda \in \Lambda^{+}(r+(n-1) s)$ so $\sum_{i=1}^{s} \lambda_{i} \geq(n-1) s$. Proof. Given a rational $(\rho, \sigma)$-tableau $(\mathfrak{r}, \mathfrak{s})$, we construct a $\lambda$-tableau $\mathfrak{t}$ as follows. Draw a rectangular diagram with $s$ rows and $n$ columns. Rotate the tableau $\mathfrak{s}$ by 180 degrees, and place it in the bottom right corner of the rectangle. Place the tableau $\mathfrak{r}$ on the left side below the rectangle. Fill the empty boxes of the rectangle with numbers such that in each row the entries that do not appear in $\mathfrak{t}$ appear in the empty boxes in increasing order. Let $t$ be the tableau consisting of the formerly empty boxes and the boxes of $\mathfrak{r}$. We illustrate this procedure with an example. Let $n=5, r=4, s=5$ and $k=1$, and let

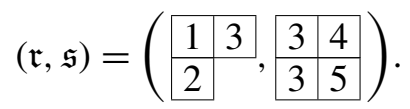

Then

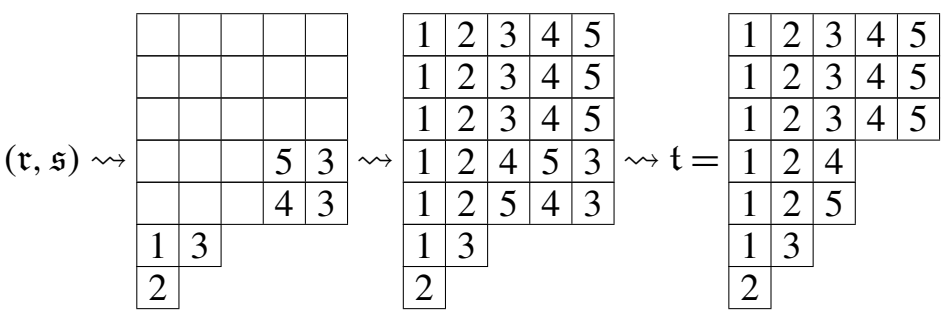

It is now easy to give an inverse. Just draw the rectangle into the tableau $\mathfrak{t}$, fill the empty boxes of the rectangle in a similar way as before, and rotate these back to obtain $\mathfrak{s}$. Note $\mathfrak{r}$ is the part of the tableau $\mathfrak{t}$ that lies outside the rectangle. We have to show that these bijections provide standard tableaux of the right shape. 
Suppose $(\mathfrak{r}, \mathfrak{s})$ is a rational $(\rho, \sigma)$-tableau, so $\mathfrak{t}$ is a $\lambda$-tableau with $\lambda_{i}=n-\sigma_{s+1-i}$ for $i \leq s$ and $\lambda_{i}=\rho_{i-s}$ for $i>s$. So $\lambda_{i} \geq \lambda_{i+1}$ for $i<s$ is equivalent to $\sigma_{s+1-i} \leq \sigma_{s-i}$, and for $i>s$ it is equivalent to $\rho_{i-s} \geq \rho_{i+1-s}$. Now $\rho_{1}+\sigma_{1}=\lambda_{s+1}-\left(\lambda_{s}-n\right)$. This shows that $\lambda$ is a partition if and only if $\rho$ and $\sigma$ are partitions with $\rho_{1}+\sigma_{1} \leq n$. We still have to show that $(\mathfrak{r}, \mathfrak{s})$ is standard if and only if $\mathfrak{t}$ is standard.

By definition, all standard tableaux have increasing rows. A tableau has nondecreasing columns if and only if for all $i=1, \ldots, n$ and all rows (except for the last row) the number of entries at most $i$ in this row is greater than or equal to the number of entries at most $i$ in the next row. Now it follows from the construction that $t$ has nondecreasing columns inside the rectangle if and only if $\mathfrak{s}$ has nondecreasing columns, $\mathfrak{t}$ has nondecreasing columns outside the rectangle if and only if $\mathfrak{r}$ has nondecreasing columns and the columns in $\mathfrak{t}$ do not decrease from row $s$ to row $s+1$ if and only if condition (6.1.1) holds.

Definition 6.4. Let $\mathfrak{d e t}_{q}^{(k)} \in A_{q}(n ; k, k)$ with $k \geq 1$ be recursively defined by $\mathfrak{d e t}_{q}^{(1)}:=\sum_{l=1}^{n} x_{1 l} x_{1 l}^{*}$ and $\mathfrak{d e t}_{q}^{(k)}:=\sum_{l=1}^{n} x_{1 l} \mathfrak{d e t}_{q}^{(k-1)} x_{1 l}^{*}$ for $k>1$.

Let a (rational) bideterminant $\left((\mathfrak{r}, \mathfrak{s}) \mid\left(\mathfrak{r}^{\prime}, \mathfrak{s}^{\prime}\right)\right) \in A_{q}(n ; r, s)$ be defined by

$$
\left((\mathfrak{r}, \mathfrak{s}) \mid\left(\mathfrak{r}^{\prime}, \mathfrak{s}^{\prime}\right)\right):=\left(\mathfrak{r} \mid \mathfrak{r}^{\prime}\right) \mathfrak{d e t} \mathfrak{t}_{q}^{(k)}\left(\mathfrak{s} \mid \mathfrak{s}^{\prime}\right)^{*}
$$

whenever $\left[(\mathfrak{r}, \mathfrak{s}),\left(\mathfrak{r}^{\prime}, \mathfrak{s}^{\prime}\right)\right]$ is a rational $(\rho, \sigma)$-bitableau such that $\rho \in \Lambda^{+}(r-k)$ and $\sigma \in \Lambda^{+}(s-k)$ for some $k=0,1, \ldots, \min (r, s)$.

Note that the proof of Lemma 5.1 and Remark 3.3(4) show that $\iota\left(\mathfrak{d e t}_{q}^{(k)}\right)=\operatorname{det}_{q}^{k}$. Furthermore, if $\rho_{1}$ or $\sigma_{1}>n$, then the bideterminant of a $(\rho, \sigma)$-bitableau vanishes. As a direct consequence of Theorem 5.3, we get the following:

Lemma 6.5. Let $(\mathfrak{r}, \mathfrak{s})$ and $\left(\mathfrak{r}^{\prime}, \mathfrak{s}^{\prime}\right)$ be two standard rational tableaux, and let $\mathfrak{t}$ and $\mathfrak{t}^{\prime}$ be the (standard) tableaux obtained from the correspondence of Lemma 6.3. Then

$$
\iota\left((\mathfrak{r}, \mathfrak{s}) \mid\left(\mathfrak{r}^{\prime}, \mathfrak{s}^{\prime}\right)\right)=(-q)^{c\left(\mathfrak{t}, \mathfrak{t}^{\prime}\right)}\left(\mathfrak{t} \mid \mathfrak{t}^{\prime}\right)
$$

for some integer $c\left(t, \mathfrak{t}^{\prime}\right)$. In particular, the bideterminants of standard rational bitableaux are linearly independent.

Proof. This follows directly from Theorem 5.3, the construction of the bijection and $\iota\left(\mathfrak{d e t}_{q}^{(k)}\right)=\operatorname{det}_{q}^{k}$. The second statement follows from the fact that the $\left(\mathfrak{t} \mid \mathfrak{t}^{\prime}\right) \mathrm{s}$ are linearly independent.

Lemma 6.6. We have

$$
\begin{array}{cc}
\sum_{l=1}^{n} x_{i l} \mathfrak{d e t}_{q}^{(k)} x_{j l}^{*}=0 & \text { for } i \neq j, \\
\sum_{l=1}^{n} q^{2 l} x_{l i} \mathfrak{d e t}_{q}^{(k)} x_{l j}^{*}=0 & \text { for } i \neq j,
\end{array}
$$


and

$$
\sum_{l=1}^{n} q^{2 l-2 i} x_{l i} \mathfrak{d e t}_{q}^{(k)} x_{l i}^{*}=\sum_{l=1}^{n} x_{j l} \mathfrak{d e t}_{q}^{(k)} x_{j l}^{*} .
$$

Proof. Without loss of generality, we may assume $k=1$. Suppose that $i, j \neq 1$. Then

$$
\begin{aligned}
\sum_{l=1}^{n} x_{i l} \mathfrak{d e t}_{q}^{(1)} x_{j l}^{*}= & \sum_{k, l=1}^{n} x_{i k} x_{1 l} x_{1 l}^{*} x_{j k}^{*}=\sum_{k<l} x_{1 l} x_{i k} x_{j k}^{*} x_{1 l}^{*}+q^{-2} \sum_{k} x_{1 k} x_{i k} x_{j k}^{*} x_{1 k}^{*} \\
& +\sum_{k>l}\left(x_{1 l} x_{i k} x_{j k}^{*} x_{1 l}^{*}+\left(q^{-1}-q\right)\left(x_{1 k} x_{i l} x_{1 l}^{*} x_{j k}^{*}+x_{1 l} x_{i k} x_{1 k}^{*} x_{j l}^{*}\right)\right) \\
= & \sum_{k, l} x_{1 l} x_{i k} x_{j k}^{*} x_{1 l}^{*}+\left(q^{-2}-1\right) \sum_{k} q x_{1 k} x_{i k} x_{1 k}^{*} x_{j k}^{*} \\
& +\left(q^{-1}-q\right) \sum_{k>l}\left(x_{1 k} x_{i l} x_{1 l}^{*} x_{j k}^{*}+x_{1 l} x_{i k} x_{1 k}^{*} x_{j l}^{*}\right) \\
= & \delta_{i j} \mathfrak{d e t}_{q}^{(2)}+\left(q^{-1}-q\right) \sum_{k, l} x_{1 k} x_{i l} x_{1 l}^{*} x_{j k}^{*}=\delta_{i j} \mathfrak{d e t} \mathfrak{t}_{q}^{(2)} .
\end{aligned}
$$

For $j \neq 1$, we have

$$
\begin{aligned}
\sum_{l=1}^{n} x_{1 l} \mathfrak{d e t}_{q}^{(1)} x_{j l}^{*}= & \sum_{k, l=1}^{n} x_{1 k} x_{1 l} x_{1 l}^{*} x_{j k}^{*}=\sum_{k<l} q x_{1 l} x_{1 k} x_{j k}^{*} x_{1 l}^{*}+q^{-1} \sum_{k} x_{1 k} x_{1 k} x_{j k}^{*} x_{1 k}^{*} \\
& +\sum_{k>l}\left(q^{-1} x_{1 l} x_{1 k} x_{j k}^{*} x_{1 l}^{*}+\left(q^{-1}-q\right) x_{1 k} x_{1 l} x_{j l}^{*} x_{1 k}^{*}\right) \\
= & \sum_{k, l} q^{-1} x_{1 l} x_{1 k} x_{j k}^{*} x_{1 l}^{*}=0 .
\end{aligned}
$$

Similarly, one can show that

$$
\begin{gathered}
\sum_{l=1}^{n} x_{i l} \mathfrak{d e t}_{q}^{(1)} x_{1 l}^{*}=0 \quad \text { for } i \neq 1, \\
\sum_{l=1}^{n} q^{2 l-2 i} x_{l i} \mathfrak{d e t}_{q}^{(1)} x_{l j}^{*}=\delta_{i j} \sum_{l=1}^{n} q^{2 l-2} x_{l 1} \mathfrak{d e t}_{q}^{(1)} x_{l 1}^{*} \quad \text { for } i, j \neq 1 \\
\sum_{l=1}^{n} q^{2 l-2} x_{l 1} \mathfrak{d} \mathfrak{e t}_{q}^{(1)} x_{l j}^{*}=0 \quad \text { for } j \neq 1 \\
\sum_{l=1}^{n} q^{2 l-2 i} x_{l i} \mathfrak{d e t}_{q}^{(1)} x_{l 1}^{*}=0 \quad \text { for } i \neq 1
\end{gathered}
$$

Finally, 


$$
\begin{aligned}
\sum_{l=1}^{n} q^{2 l-2} x_{l 1} \mathfrak{d e t}_{q}^{(1)} x_{l 1}^{*} & \\
= & \sum_{l, k} q^{2 l-2} x_{l 1} x_{1 k} x_{1 k}^{*} x_{l 1}^{*}=\sum_{l, k \neq 1} q^{2 l-2} x_{1 k} x_{l 1} x_{l 1}^{*} x_{1 k}^{*} \\
& \quad+\sum_{l \neq 1} q^{2 l-4} x_{11} x_{l 1} x_{l 1}^{*} x_{11}^{*}+\sum_{k \neq 1} q^{2} x_{1 k} x_{11} x_{11}^{*} x_{1 k}^{*}+x_{11} x_{11} x_{11}^{*} x_{11}^{*} \\
= & \mathfrak{d e t}_{q}^{(2)}+\sum_{l \neq 1} q^{2 l-4}\left(1-q^{2}\right) x_{11} x_{l 1} x_{l 1}^{*} x_{11}^{*}+\sum_{k \neq 1}\left(q^{2}-1\right) x_{1 k} x_{11} x_{11}^{*} x_{1 k}^{*} \\
= & \mathfrak{d e t}_{q}^{(2)}+\left(1-q^{2}\right)\left(\sum_{l \neq 1} q^{2 l-4} x_{11} x_{l 1} x_{l 1}^{*} x_{11}^{*}-q^{-2} \sum_{k \neq 1} x_{11} x_{1 k} x_{1 k}^{*} x_{11}^{*}\right) \\
= & \mathfrak{d e t}_{q}^{(2)} .
\end{aligned}
$$

Lemma 6.7. Suppose $\boldsymbol{r}=\left(r_{1}, \ldots, r_{k}\right), s=\left(s_{1}, \ldots, s_{k}\right) \in I(n, k)$ are fixed. Let $j \in\{1, \ldots, n\}$ and $k \geq 1$. Then we have, modulo $\mathfrak{d e t}_{q}^{(1)}$,

$$
\begin{gathered}
\sum_{j<j_{1}<j_{2}<\cdots<j_{k}}\left(\boldsymbol{r} \mid j_{k} \ldots j_{2} j_{1}\right)_{r}\left(\boldsymbol{s} \mid j_{1} j_{2} \ldots j_{k}\right)_{r}^{*} \\
\equiv(-1)^{k} q^{2 \sum_{i=0}^{k-1} i} \sum_{j_{1}<j_{2}<\cdots<j_{k} \leq j}\left(\boldsymbol{r} \mid j_{k} \ldots j_{2} j_{1}\right)_{r}\left(\boldsymbol{s} \mid j_{1} j_{2} \ldots j_{k}\right)_{r}^{*} .
\end{gathered}
$$

Proof. The only difference between $\left(s \mid j_{1} j_{2} \ldots j_{k}\right)_{r}^{*}$ and $\left(s \mid j_{1} j_{2} \ldots j_{k}\right)_{l}^{*}$ is on a power of $-q$ not depending on $j_{1}, j_{2}, \ldots, j_{k}$. Thus, we can show the lemma with $(\cdot, \cdot)_{r}^{*}$ replaced by $(\cdot, \cdot)_{l}^{*}$. Similarly, we can assume that $r_{1}<r_{2}<\cdots<r_{k}$ and $s_{1}>s_{2}>\cdots>s_{k}$. Note that, modulo $\mathfrak{d e t}_{q}^{(1)}$, we have the relations $\sum_{k=1}^{n} x_{i k} x_{j k}^{*} \equiv 0$. It follows that the lemma is true for $k=1$. Assume that the lemma holds for $k-1$. If $M$ is an ordered set, let $M^{k,<}$ be the set of $k$-tuples in $M$ with increasing entries. For a subset $M \subset\{1, \ldots, n\}$, we have

$$
\begin{aligned}
\sum_{\boldsymbol{j} \in M^{k,<}}\left(\boldsymbol{r} \mid j_{k} \ldots j_{2} j_{1}\right)_{r}\left(\boldsymbol{s} \mid j_{1} j_{2} \ldots j_{k}\right)_{l}^{*} & \\
& =\sum_{\boldsymbol{j} \in M^{k,<}, w}(-q)^{-l(w)}\left(\boldsymbol{r} \mid j_{k} \ldots j_{2} j_{1}\right)_{r} x_{s_{1} j_{w 1}}^{*} \cdots x_{s_{k} j_{w k}}^{*} \\
& =\sum_{\boldsymbol{j} \in M^{k,<}, w}\left(\boldsymbol{r} \mid j_{w k} \ldots j_{w 1}\right)_{r} x_{s_{1} j_{w 1}}^{*} \cdots x_{s_{k} j_{w k}}^{*} \\
& =\sum_{\boldsymbol{j} \in M^{k}}\left(\boldsymbol{r} \mid j_{k} \cdots j_{1}\right)_{r} x_{s_{1} j_{1}}^{*} \cdots x_{s_{k} j_{k}}^{*} .
\end{aligned}
$$

Applying Laplace's expansion, we can write a quantum minor $\left(\boldsymbol{r} \mid \boldsymbol{j}_{1} \boldsymbol{j}_{2}\right)_{r}$ as a linear 
combination of products of quantum minors, say

$$
\left(\boldsymbol{r} \mid \boldsymbol{j}_{1} \boldsymbol{j}_{2}\right)_{r}=\sum_{l} c_{l}\left(\boldsymbol{r}_{l}^{\prime} \mid \boldsymbol{j}_{1}\right)_{r}\left(\boldsymbol{r}_{l}^{\prime \prime} \mid \boldsymbol{j}_{2}\right)_{r}
$$

Then with $\epsilon_{k}:=(-1)^{k} q^{2 \sum_{i=0}^{k-1} i}, \boldsymbol{j}=\left(j_{1}, \ldots, j_{k}\right), \boldsymbol{j}^{\prime}=\left(j_{1}, \ldots, j_{k-1}\right), C=\{1 \ldots j\}$ and $D=\{j+1 \ldots n\}$, we have

$$
\begin{aligned}
& \sum_{\boldsymbol{j} \in D^{k,<}}\left(\boldsymbol{r} \mid j_{k} \cdots j_{2} j_{1}\right)_{r}\left(\boldsymbol{s} \mid j_{1} j_{2} \ldots j_{k}\right)_{l}^{*}=\sum_{\boldsymbol{j} \in D^{k}}\left(\boldsymbol{r} \mid j_{k} \cdots j_{1}\right)_{r} x_{s_{1} j_{1}}^{*} \cdots x_{s_{k} j_{k}}^{*} \\
& =\sum_{j \in D^{k}, l} c_{l}\left(\boldsymbol{r}_{l}^{\prime} \mid j_{k}\right)_{r}\left(\boldsymbol{r}_{l}^{\prime \prime} \mid j_{k-1} \cdots j_{1}\right)_{r} x_{s_{1} j_{1}}^{*} \cdots x_{s_{k-1} j_{k-1}}^{*} x_{s_{k} j_{k}}^{*} \\
& \equiv \epsilon_{k-1} \sum_{j^{\prime} \in C^{k-1}, l, j_{k}>j} c_{l}\left(\boldsymbol{r}_{l}^{\prime} \mid j_{k}\right)_{r}\left(\boldsymbol{r}_{l}^{\prime \prime} \mid j_{k-1} \cdots j_{1}\right)_{r} x_{s_{1} j_{1}}^{*} \cdots x_{s_{k-1} j_{k-1}}^{*} x_{s_{k} j_{k}}^{*} \\
& =\epsilon_{k-1} \sum_{j^{\prime} \in C^{k-1}, j_{k}>j}\left(\boldsymbol{r} \mid j_{k} j_{k-1} \cdots j_{1}\right)_{r} x_{s_{1} j_{1}}^{*} \cdots x_{s_{k-1} j_{k-1}}^{*} x_{s_{k} j_{k}}^{*} \\
& =\epsilon_{k-1} \sum_{\boldsymbol{j}^{\prime} \in C^{k-1}, j_{k}>j}(-q)^{k-1}\left(\boldsymbol{r} \mid j_{k-1} \cdots j_{1} j_{k}\right)_{r} x_{s_{k} j_{k}}^{*} x_{s_{1} j_{1}}^{*} \cdots x_{s_{k-1} j_{k-1}}^{*} \\
& =\epsilon_{k-1} \sum_{\boldsymbol{j}^{\prime} \in C^{k-1}, l, j_{k}>j}(-q)^{k-1} c_{l}\left(\boldsymbol{r}_{l}^{\prime} \mid j_{k-1} \cdots j_{1}\right)_{r} x_{\boldsymbol{r}_{l}^{\prime \prime} j_{k}} x_{s_{k} j_{k}}^{*} x_{s_{1} j_{1}}^{*} \cdots x_{s_{k-1} j_{k-1}}^{*} \\
& \equiv-\epsilon_{k-1} \sum_{j \in C^{k}, l}(-q)^{k-1} c_{l}\left(\boldsymbol{r}_{l}^{\prime} \mid j_{k-1} \cdots j_{1}\right)_{r} x_{\boldsymbol{r}_{l}^{\prime \prime} j_{k}} x_{s_{k} j_{k}}^{*} x_{s_{1} j_{1}}^{*} \cdots x_{s_{k-1} j_{k-1}}^{*} \\
& =-\epsilon_{k-1} \sum_{j \in C^{k}}(-q)^{k-1}\left(\boldsymbol{r} \mid j_{k-1} \cdots j_{1} j_{k}\right)_{r} x_{s_{k} j_{k}}^{*} x_{s_{1} j_{1}}^{*} \cdots x_{s_{k-1} j_{k-1}}^{*} \\
& =-\epsilon_{k-1} \sum_{j \in C^{k,<}}(-q)^{k-1}\left(\boldsymbol{r} \mid j_{k} \ldots j_{1}\right)_{r}\left(s_{k} s_{1} \ldots s_{k-1} \mid j_{1} \ldots j_{k}\right)_{l}^{*} \\
& =-\epsilon_{k-1} \sum_{j \in C^{k,<}}(-q)^{2(k-1)}\left(\boldsymbol{r} \mid j_{k} \ldots j_{1}\right)_{r}\left(s_{1} \ldots s_{k} \mid j_{1} \ldots j_{k}\right)_{l}^{*} \\
& =\epsilon_{k} \sum_{\boldsymbol{j} \in C^{k,<}}\left(\boldsymbol{r} \mid j_{k} \ldots j_{2} j_{1}\right)_{r}\left(\boldsymbol{s} \mid j_{1} j_{2} \ldots j_{k}\right)_{l}^{*} \text {. }
\end{aligned}
$$

Lemma 6.8. Let $\boldsymbol{r}^{\prime}$ and $\boldsymbol{s}^{\prime}$ be strictly increasing multi-indices considered as tableaux with one row. Let $i$ be the maximal entry appearing, and suppose that $i$ is minimal such that $i$ violates condition (6.1.1). Let I be the set of entries appearing in both $\boldsymbol{r}^{\prime}$ and $\boldsymbol{s}^{\prime}$; then we have $i \in I$. Let $L_{1}:=\left\{k_{1}, \ldots, k_{l_{1}}\right\}$ be the set of entries of $\boldsymbol{r}^{\prime}$ not appearing in $\boldsymbol{s}^{\prime}$, let $L_{2}:=\left\{k_{1}^{\prime}, \ldots, k_{l_{2}}^{\prime}\right\}$ be the set of entries of $\boldsymbol{s}^{\prime}$ not appearing in $\boldsymbol{r}^{\prime}$, and let $i_{1}<i_{2}<\cdots<i_{k}=i$ be the entries of $I$. 
Let $D:=\left\{i_{1}, \ldots, i_{k}, i_{k}+1, i_{k}+2, \ldots, n\right\}$ and $C:=\{1, \ldots, n\} \backslash\left(D \cup L_{1} \cup L_{2}\right)$. Furthermore, for $j_{1}, \ldots, j_{t} \in\{1, \ldots, n\}$, let

$$
m\left(j_{1}, \ldots, j_{t}\right):=\left|\left\{(l, c) \in\{1, \ldots, t\} \times C: j_{l}<c\right\}\right| .
$$

Let $\boldsymbol{k}:=\left(k_{1}, \ldots, k_{l_{1}}\right)$ and $\boldsymbol{k}^{\prime}:=\left(k_{1}^{\prime}, \ldots, k_{l_{2}}^{\prime}\right)$, and let $\boldsymbol{r}$ and $\boldsymbol{s}$ be multi-indices of the same length as $\boldsymbol{r}^{\prime}$ (resp. $\boldsymbol{s}^{\prime}$ ); then we have

$$
\sum_{\boldsymbol{j} \in D^{k,<}} q^{2 m(\boldsymbol{j})}\left(\boldsymbol{r} \mid \boldsymbol{k} j_{k} \ldots j_{1}\right)_{r}\left(\boldsymbol{s} \mid j_{1} \ldots j_{k} \boldsymbol{k}^{\prime}\right)_{r}^{*} \equiv 0 \bmod \mathfrak{d e t} \mathfrak{t}_{q}^{(1)} .
$$

Proof. Note that $i \in I$ and $i=2 k+l_{1}+l_{2}-1$; otherwise, $i-1$ would violate (6.1.1). Therefore, $|C|=k-1$. Let $c_{\max }$ be the maximal element of $C, \tilde{C}=\left\{1, \ldots, c_{\max }\right\}$, $\tilde{D}=\left\{c_{\max }+1, c_{\max }+2, \ldots, n\right\} \subset D \cup L_{1} \cup L_{2}, D_{-}=\left\{d \in D: d<c_{\max }\right\}$ and $D_{+}=\left\{d \in D: d>c_{\max }\right\}$. With $\tilde{\boldsymbol{j}}=\left(j_{1}, \ldots, j_{l}\right)$ and $\widehat{\boldsymbol{j}}=\left(j_{l+1}, \ldots, j_{k}\right)$, we have

$$
\begin{aligned}
& \sum_{\boldsymbol{j} \in D^{k,<}} q^{2 m(\boldsymbol{j})}\left(\boldsymbol{r} \mid \boldsymbol{k} j_{k} \ldots j_{1}\right)_{r}\left(\boldsymbol{s} \mid j_{1} \ldots j_{k} \boldsymbol{k}^{\prime}\right)_{r}^{*} \\
&=\sum_{l=0}^{k} \sum_{\tilde{\boldsymbol{j}} \in D_{-}^{l,<}} q^{2 m(\tilde{\boldsymbol{j}})} \sum_{\hat{\boldsymbol{j}} \in D_{+}^{k-l,<}}\left(\boldsymbol{r} \mid \boldsymbol{k} j_{k} \ldots j_{1}\right)_{r}\left(\boldsymbol{s} \mid j_{1} \ldots j_{k} \boldsymbol{k}^{\prime}\right)_{r}^{*} .
\end{aligned}
$$

Without loss of generality, we may assume that the entries in $s$ are increasing. We apply Laplace's expansion and Lemma 6.7 to get for fixed $l$ and $\tilde{\boldsymbol{j}}$

$$
\begin{aligned}
\sum_{\widehat{\boldsymbol{j}} \in D_{+}^{k-l,<}} & \left(\boldsymbol{r} \mid \boldsymbol{k} j_{k} \ldots j_{1}\right)_{r}\left(\boldsymbol{s} \mid j_{1} \ldots j_{k} \boldsymbol{k}^{\prime}\right)_{r}^{*}=\sum_{\widehat{\boldsymbol{j}} \in \tilde{D}^{k-l,<}}\left(\boldsymbol{r} \mid \boldsymbol{k} j_{k} \ldots j_{1}\right)_{r}\left(\boldsymbol{s} \mid j_{1} \ldots j_{k} \boldsymbol{k}^{\prime}\right)_{r}^{*} \\
= & q^{2 l(k-l)} \sum_{\widehat{\boldsymbol{j}} \in \tilde{D}^{k-l,<}}\left(\boldsymbol{r} \mid \boldsymbol{k} j_{l} \ldots j_{1} j_{k} \ldots j_{l+1}\right)_{r}\left(\boldsymbol{s} \mid j_{l+1} \ldots j_{k} j_{1} \ldots j_{l} \boldsymbol{k}^{\prime}\right)_{r}^{*} \\
\equiv & \epsilon_{k-l} q^{2 l(k-l)} \sum_{\widehat{\boldsymbol{j}} \in \tilde{C}^{k-l,<}}\left(\boldsymbol{r} \mid \boldsymbol{k} j_{l} \ldots j_{1} j_{k} \ldots j_{l+1}\right)_{r}\left(\boldsymbol{s} \mid j_{l+1} \ldots j_{k} j_{1} \ldots j_{l} \boldsymbol{k}^{\prime}\right)_{r}^{*} \\
= & \epsilon_{k-l} q^{2 l(k-l)} \sum_{\widehat{\boldsymbol{j}} \in\left(C \cup D_{-}\right)^{k-l,<}}\left(\boldsymbol{r} \mid \boldsymbol{k} j_{l} \ldots j_{1} j_{k} \ldots j_{l+1}\right)_{r}\left(\boldsymbol{s} \mid j_{l+1} \ldots j_{k} j_{1} \ldots j_{l} \boldsymbol{k}^{\prime}\right)_{r}^{*}
\end{aligned}
$$

This expression can be substituted into (6.8.1). Each nonzero summand belongs to a disjoint union $S_{1} \cup \dot{\cup} S_{2}=S \subset C \cup D_{-}$such that $|S|=k, S_{1}=\left\{j_{1}, \ldots, j_{l}\right\}$ and $S_{2}=\left\{j_{l+1}, \ldots, j_{k}\right\}$. We will show that the summands belonging to some fixed set $S$ cancel out.

Therefore, we claim that for each subset $S \subset C \cup D_{-}$with $k$ elements, there exists some $d \in D \cap S$ such that $m(d)=|\{s \in S: s>d\}|$. Suppose not. Since $|C|=k-1$, $S$ contains at least one element of $D$. Let $s_{1}<s_{2}<\cdots<s_{m}$ be the elements of $D \cap S$. We show by downward induction that $m\left(s_{l}\right)>\left|\left\{s \in S: s>s_{l}\right\}\right|$ for $1 \leq l \leq m$; $m\left(s_{m}\right)$ is the cardinality of $\left\{s_{m}+1, \ldots, c_{\max }\right\} \cap C$. Since all $s \in S$ with $s>s_{m}$ are 
elements of $C$, we have $\left\{s_{m}+1, \ldots, c_{\max }\right\} \cap S \subset\left\{s_{m}+1, \ldots, c_{\max }\right\} \cap C$, and thus, $m\left(s_{m}\right) \geq\left|\left\{s \in S: s>s_{m}\right\}\right|$. By assumption, we have $>$ instead of $\geq$. Now suppose $m\left(s_{l}\right)>\left|\left\{s \in S: s>s_{l}\right\}\right|$, so $\left\{s \in S: s_{l-1}<s \leq s_{l}\right\}=\left\{s \in S \cap C: s_{l-1}<s<s_{l}\right\} \cup\left\{s_{l}\right\}$; thus, $S$ contains at most $m\left(s_{l-1}\right)-m\left(s_{l}\right)$ elements between $s_{l-1}$ and $s_{l}$, so at most $m\left(s_{l-1}\right)-m\left(s_{l}\right)+1+m\left(s_{l}\right)-1=m\left(s_{l-1}\right)$ elements are greater than $s_{l-1}$. By assumption, we have $m\left(s_{l-1}\right)>\left|\left\{s \in S: s>s_{l-1}\right\}\right|$. We have shown that $S$ contains less than $m\left(s_{1}\right)$ elements greater than $s_{1}$; thus, $S$ contains less than $|C|+1=k$ elements, which is a contradiction. This shows the claim.

Let $S \subset C \cup D_{-}$be fixed subset of cardinality $k$. By the previous consideration, there is an element $d \in D \cap S$ with $m(d)=|\{s \in S: s>d\}|$. We claim that the summand for $S_{1}$ and $S_{2}$ with $d \in S_{1}$ cancels the summand for $S_{1} \backslash\{d\}$ and $S_{2} \cup\{d\}$. Note that

$$
\begin{aligned}
\left(\boldsymbol{r} \mid \boldsymbol{k} j_{l} \ldots \widehat{d}\right. & \left.\ldots j_{1} j_{k} \ldots d \ldots j_{l+1}\right)_{r}\left(\boldsymbol{s} \mid j_{l+1} \ldots d \ldots j_{k} j_{1} \ldots \widehat{d} \ldots j_{l} \boldsymbol{k}^{\prime}\right)_{r}^{*} \\
& =q^{2|\{s \in S: s>d\}|-2(l-1)}\left(\boldsymbol{r} \mid \boldsymbol{k} j_{l} \ldots j_{1} j_{k} \ldots j_{l+1}\right)_{r}\left(\boldsymbol{s} \mid j_{l+1} \ldots j_{k} j_{1} \ldots j_{l} \boldsymbol{k}^{\prime}\right)_{r}^{*} .
\end{aligned}
$$

Comparing coefficients, we see that both summands cancel.

Theorem 6.9 (Rational Straightening Algorithm). The set of bideterminants of standard rational bitableaux forms an $R$-basis of $A_{q}(n ; r, s)$.

Proof. We have to show that the bideterminants of standard rational bitableaux generate $A_{q}(n ; r, s)$. Clearly, the bideterminants $\left((\mathfrak{r}, \mathfrak{s}) \mid\left(\mathfrak{r}^{\prime}, \mathfrak{s}^{\prime}\right)\right)$ with $\mathfrak{r}, \mathfrak{r}^{\prime}, \mathfrak{s}$ and $\mathfrak{s}^{\prime}$ standard tableaux generate $A_{q}(n ; r, s)$. Let $\operatorname{cont}(\mathfrak{r})(\operatorname{resp}$. cont $(\mathfrak{s}))$ be the content of $\mathfrak{r}$ (resp. $\mathfrak{s})$ defined in Definition 3.4.

Let $\mathfrak{r}, \mathfrak{r}^{\prime}, \mathfrak{s}$ and $\mathfrak{s}^{\prime}$ be standard tableaux, and suppose that the rational bitableau $\left[(\mathfrak{r}, \mathfrak{s}),\left(\mathfrak{r}^{\prime}, \mathfrak{s}^{\prime}\right)\right]$ is not standard. It suffices to show the bideterminant $\left((\mathfrak{r}, \mathfrak{s}) \mid\left(\mathfrak{r}^{\prime}, \mathfrak{s}^{\prime}\right)\right)$ is a linear combination of bideterminants $\left.(\widehat{\mathfrak{r}}, \widehat{\mathfrak{s}}) \mid\left(\widehat{\mathfrak{r}}^{\prime}, \widehat{\mathfrak{s}}^{\prime}\right)\right)$ such that $\widehat{\mathfrak{r}}$ has fewer boxes than $\mathfrak{r}$ or $\operatorname{cont}(\mathfrak{r})>\operatorname{cont}(\widehat{\mathfrak{r}}) \operatorname{or} \operatorname{cont}(\mathfrak{s})>\operatorname{cont}(\widehat{\mathfrak{s}})$ in the lexicographical order. Without loss of generality, we make the following assumptions:

- In the nonstandard rational bitableau $\left[(\mathfrak{r}, \mathfrak{s}),\left(\mathfrak{r}^{\prime}, \mathfrak{s}^{\prime}\right)\right]$, the rational tableau $\left(\mathfrak{r}^{\prime}, \mathfrak{s}^{\prime}\right)$ is nonstandard. Note that the automorphism of Remark 4.2 maps a bideterminant $\left((\mathfrak{r}, \mathfrak{s}) \mid\left(\mathfrak{r}^{\prime}, \mathfrak{s}^{\prime}\right)\right)$ to the bideterminant $\left(\left(\mathfrak{r}^{\prime}, \mathfrak{s}^{\prime}\right) \mid(\mathfrak{r}, \mathfrak{s})\right)$.

- Suppose that $(\mathfrak{r}, \mathfrak{s})$ and $\left(\mathfrak{r}^{\prime}, \mathfrak{s}^{\prime}\right)$ are $(\rho, \sigma)$-tableaux. In view of Lemma 6.6, we can assume that $\rho \in \Lambda^{+}(r)$ and $\sigma \in \Lambda^{+}(s)$.

- The tableaux $\mathfrak{r}, \mathfrak{r}^{\prime}, \mathfrak{s}$ and $\mathfrak{s}^{\prime}$ have only one row (each bideterminant has a factor of this type), and we can use Theorem 3.5 to write nonstandard bideterminants as a linear combination of standard ones of the same content.

- Let $i$ be minimal such that condition (6.1.1) of Definition 6.1 is violated for $i$. Applying Laplace's expansion, we may assume that there is no greater entry than $i$ in $\mathfrak{r}^{\prime}$ and in $\mathfrak{s}^{\prime}$. 
Note that all elements of $A_{q}(n ; r, s)$ having a factor $\mathfrak{d e t}_{q}^{(1)}$ can be written as a linear combination of bideterminants of rational $(\rho, \sigma)$-bitableaux with $\rho \in \Lambda^{+}(r-k)$, $k>0$. Thus, it suffices to show that $\left((\mathfrak{r}, \mathfrak{s}) \mid\left(\mathfrak{r}^{\prime}, \mathfrak{s}^{\prime}\right)\right)$ is, modulo $\mathfrak{d e t}_{q}^{(1)}$, a linear combination of bideterminants of "lower content". The summand of highest content in Lemma 6.8 is that one for $\boldsymbol{j}=\left(i_{1}, i_{2}, \ldots, i_{k}\right)$, and this summand is a scalar multiple (a power of $-q$, which is invertible) of $\left((\mathfrak{r}, \mathfrak{s}) \mid\left(\mathfrak{r}^{\prime}, \mathfrak{s}^{\prime}\right)\right.$ ).

The following is an immediate consequence of the preceding theorem and Lemma 6.3.

Corollary 6.10. There exists an R-linear map $\phi: A_{q}(n, r+(n-1) s) \rightarrow A_{q}(n ; r, s)$ given on a basis by $\phi\left(\mathfrak{t} \mid \mathfrak{t}^{\prime}\right):=(-q)^{-c\left(\mathfrak{t}, \mathfrak{t}^{\prime}\right)}\left((\mathfrak{r}, \mathfrak{s}) \mid\left(\mathfrak{r}^{\prime}, \mathfrak{s}^{\prime}\right)\right)$ if the shape $\lambda$ of $\mathfrak{t}$ satisfies $\sum_{i=1}^{s} \lambda_{i} \geq(n-1) s$, where $(\mathfrak{r}, \mathfrak{s})$ and $\left(\mathfrak{r}^{\prime}, \mathfrak{s}^{\prime}\right)$ are the rational tableaux respectively corresponding to $\mathfrak{t}$ and $\mathfrak{t}^{\prime}$ under the correspondence of Lemma 6.3, and $\phi\left(\mathfrak{t} \mid \mathfrak{t}^{\prime}\right):=0$ otherwise. We have

$$
\phi \circ \iota=\operatorname{id}_{A_{q}(n ; r, s)},
$$

and thus, $\pi=\iota^{*}$ is surjective.

As noted in Section 2, we now have the main result.

Theorem 6.11 (Schur-Weyl duality for mixed tensor space, II). We have

$$
S_{q}(n ; r, s)=\operatorname{End}_{\mathfrak{B}_{r, s}(q)}\left(V^{\otimes r} \otimes V^{* \otimes s}\right)=\rho_{\mathrm{mxd}}(\mathbf{U})=\rho_{\mathrm{mxd}}\left(\mathbf{U}^{\prime}\right),
$$

and $S_{q}(n ; r, s)$ is $R$-free with a basis indexed by standard rational bitableau.

Proof. The first assertion follows from the surjectivity of $\pi$; the second assertion is obtained by dualizing the basis of $A_{q}(n ; r, s)$.

\section{References}

[Benkart et al. 1994] G. Benkart, M. Chakrabarti, T. Halverson, R. Leduc, C. Lee, and J. Stroomer, "Tensor product representations of general linear groups and their connections with Brauer algebras", J. Algebra 166:3 (1994), 529-567. MR 95d:20071 Zbl 0815.20028

[Brundan and Stroppel 2011] J. Brundan and C. Stroppel, "Gradings on walled Brauer algebras and Khovanov's arc algebras”, preprint, 2011. arXiv 1107.0999

[Dipper and Donkin 1991] R. Dipper and S. Donkin, "Quantum GL ${ }_{n}$ ", Proc. London Math. Soc. (3) 63:1 (1991), 165-211. MR 92g:16055 Zbl 0734.20018

[Dipper and Doty 2008] R. Dipper and S. Doty, "The rational Schur algebra", Represent. Theory 12 (2008), 58-82. MR 2009e:20097 Zbl 1185.20052

[Dipper and James 1989] R. Dipper and G. James, "The $q$-Schur algebra", Proc. London Math. Soc.

(3) 59:1 (1989), 23-50. MR 90g:16026 Zbl 0711.20007

[Dipper et al. 2012] R. Dipper, S. Doty, and F. Stoll, "The quantized walled Brauer algebra and mixed tensor space", preprint, 2012. arXiv 0806.0264

[Goodearl 2006] K. R. Goodearl, “Commutation relations for arbitrary quantum minors”, Pacific J. Math. 228:1 (2006), 63-102. MR 2007j:17019 Zbl 1125.16034 
[Green 1996] R. M. Green, " $q$-Schur algebras as quotients of quantized enveloping algebras", $J$. Algebra 185:3 (1996), 660-687. MR 97k:17016 Zbl 0862.17007

[Hong and Kang 2002] J. Hong and S.-J. Kang, Introduction to quantum groups and crystal bases, Graduate Studies in Mathematics 42, American Mathematical Society, Providence, RI, 2002. MR 2002m:17012 Zbl 1134.17007

[Huang and Zhang 1993] R. Q. Huang and J. J. Zhang, "Standard basis theorem for quantum linear groups", Adv. Math. 102:2 (1993), 202-229. MR 94j:16067 Zbl 0793.05143

[Jantzen 1996] J. C. Jantzen, Lectures on quantum groups, Graduate Studies in Mathematics 6, American Mathematical Society, Providence, RI, 1996. MR 96m:17029 Zbl 0842.17012

[Koike 1989] K. Koike, "On the decomposition of tensor products of the representations of the classical groups: by means of the universal characters", Adv. Math. 74:1 (1989), 57-86. MR 90j:22014 Zbl 0681.20030

[Kosuda and Murakami 1993] M. Kosuda and J. Murakami, "Centralizer algebras of the mixed tensor representations of quantum group $U_{q}(\mathrm{gl}(n, \mathbf{C}))$ ", Osaka J. Math. 30:3 (1993), 475-507. MR 94k:17025 Zbl 0806.17012

[Leduc 1994] R. E. Leduc, A two-parameter version of the centralizer algebra of the mixed tensor representations of the general linear group and quantum general linear group, Ph.D. thesis, University of Wisconsin-Madison, 1994. MR 2691209

[Lusztig 1990] G. Lusztig, "Finite-dimensional Hopf algebras arising from quantized universal enveloping algebra", J. Amer. Math. Soc. 3:1 (1990), 257-296. MR 91e:17009 Zbl 0695.16006

[Schur 1927] I. Schur, "Über die rationalen Darstellungen der allgemeinen linearen Gruppe”, Sitzungsber. Akad. Berlin (1927), 58-75. Reprinted as pp. 68-85 in Gesammelte Abhandlungen, III, Springer, Berlin, 1973. MR 57 \#2858c JFM 53.0108.05

[Stembridge 1987] J. R. Stembridge, "Rational tableaux and the tensor algebra of $\mathrm{gl}_{n}$ ", J. Combin. Theory Ser. A 46:1 (1987), 79-120. MR 89a:05012 Zbl 0626.20030

[Tange 2012] R. Tange, "A bideterminant basis for a reductive monoid”, J. Pure Appl. Algebra 216:5 (2012), 1207-1221. MR 2012j:20138 Zbl 1251.05179

[Turaev 1989] V. G. Turaev, “Operator invariants of tangles, and $R$-matrices”, Izv. Akad. Nauk SSSR Ser. Mat. 53:5 (1989), 1073-1107, 1135. In Russian; translated in Math. USSR, Izv. 35:2 (1990), 411-444. MR 91e:17011 Zbl 0707.57003

Communicated by Georgia Benkart

Received 2011-11-11 Revised 2012-04-12 Accepted 2012-06-20

rdipper@mathematik.uni-stuttgart.de

Institut für Algebra und Zahlentheorie, Universität Stuttgart, Pfaffenwaldring 57, 70569 Stuttgart, Germany

doty@math.luc.edu Department of Mathematics and Statistics, Loyola University Chicago, 1023 West Sheridan Road, Chicago, IL 60660, United States

stoll@mathematik.uni-stuttgart.de

Institut für Algebra und Zahlentheorie, Universität Stuttgart, Pfaffenwaldring 57, 70569 Stuttgart, Germany 


\section{Algebra \& Number Theory}

msp.org/ant

\section{EDITORS}

MANAGING EDITOR

Bjorn Poonen

Massachusetts Institute of Technology

Cambridge, USA

\author{
EDITORIAL BOARD CHAIR \\ David Eisenbud \\ University of California \\ Berkeley, USA
}

\section{BOARD OF EDITORS}

Georgia Benkart

Dave Benson

Richard E. Borcherds

John H. Coates

J-L. Colliot-Thélène

Brian D. Conrad

Hélène Esnault

Hubert Flenner

Edward Frenkel

Andrew Granville

Joseph Gubeladze

Ehud Hrushovski

Craig Huneke

Mikhail Kapranov

Yujiro Kawamata

János Kollár

Yuri Manin

Barry Mazur

Philippe Michel
University of Wisconsin, Madison, USA

University of Aberdeen, Scotland

University of California, Berkeley, USA

University of Cambridge, UK

CNRS, Université Paris-Sud, France

University of Michigan, USA

Freie Universität Berlin, Germany

Ruhr-Universität, Germany

University of California, Berkeley, USA

Université de Montréal, Canada

San Francisco State University, USA

Hebrew University, Israel

University of Virginia, USA

Yale University, USA

University of Tokyo, Japan

Princeton University, USA

Northwestern University, USA

Harvard University, USA

École Polytechnique Fédérale de Lausanne
Susan Montgomery

Shigefumi Mori

Raman Parimala

Jonathan Pila

Victor Reiner

Karl Rubin

Peter Sarnak

Joseph H. Silverman

Michael Singer

Vasudevan Srinivas

J. Toby Stafford

Bernd Sturmfels

Richard Taylor

Ravi Vakil

Michel van den Bergh

Marie-France Vignéras

Kei-Ichi Watanabe

Efim Zelmanov
University of Southern California, USA

RIMS, Kyoto University, Japan

Emory University, USA

University of Oxford, UK

University of Minnesota, USA

University of California, Irvine, USA

Princeton University, USA

Brown University, USA

North Carolina State University, USA

Tata Inst. of Fund. Research, India

University of Michigan, USA

University of California, Berkeley, USA

Harvard University, USA

Stanford University, USA

Hasselt University, Belgium

Université Paris VII, France

Nihon University, Japan

University of California, San Diego, USA

\section{PRODUCTION}

production@msp.org

Silvio Levy, Scientific Editor

See inside back cover or msp.org/ant for submission instructions.

The subscription price for 2013 is US \$200/year for the electronic version, and \$350/year ( $\$ 40$, if shipping outside the US) for print and electronic. Subscriptions, requests for back issues and changes of subscribers address should be sent to MSP.

Algebra \& Number Theory (ISSN 1944-7833 electronic, 1937-0652 printed) at Mathematical Sciences Publishers, 798 Evans Hall \#3840, c/o University of California, Berkeley, CA 94720-3840 is published continuously online. Periodical rate postage paid at Berkeley, CA 94704, and additional mailing offices.

ANT peer review and production are managed by EditFLOW ${ }^{\circledR}$ from Mathematical Sciences Publishers.

\section{PUBLISHED BY}

- mathematical sciences publishers

nonprofit scientific publishing

http://msp.org/

(C) 2013 Mathematical Sciences Publishers 


\section{Algebra \& Number Theory}

Volume $7 \quad$ No. $5 \quad 2013$

Sharp upper bounds for the Betti numbers of a given Hilbert polynomial

GiUlio CAVIGLia and SATOShi Murai

Comparing numerical dimensions

1065

BRIAN LEHMANN

Some consequences of a formula of Mazur and Rubin for arithmetic local constants JAN NEKOVÁŘ

Quantized mixed tensor space and Schur-Weyl duality

RICHARD DIPPER, STEPHEN DOTY and FRIEDERIKE STOLL

Weakly commensurable $S$-arithmetic subgroups in almost simple algebraic groups of

types B and C

SKIP GARIBALDI and ANDREI RAPINCHUK

Minimisation and reduction of 5-coverings of elliptic curves

1179

TOM FISHER

On binary cyclotomic polynomials

1207

ÉTIENNE FOUVRY

Local and global canonical height functions for affine space regular automorphisms

SHU KAWAGUCHI

On the ranks of the 2-Selmer groups of twists of a given elliptic curve

1101

1121

147

DANIEL M. KANE 